\title{
PULSARS (THEORETICAL CONSIDERATIONS)
}

\author{
V. L. GINZBURG
}

Lebedev Physical Institute, U.S.S.R. Academy of Sciences, Moscow, U.S.S.R.

\section{Table of Contents:}

Introduction.

1. Physical Nature of Pulsars.

2. Rotating Magnetized Neutron Stars.

3. Pulsars and the Structure of Neutron Stars.

4. Electrodynamics of Rotating Magnetized Stars.

5. Mechanisms of Pulsar Radiation,

6. Some Models of Radiating Regions of Pulsars.

7. The Use of Pulsars in Astronomy and Physics.

Concluding Remarks.

Literature.

\section{Introduction}

Theorists, both physicists and astronomers, are usually greatly pleased with their choice, because to study theoretical questions is, in a sense, easier and more effective than to observe and to measure. Experimentalists and observers, on the other hand, often grumble at their fortune for their work is very labour-consuming and its success depends largely on quite non-scientific problems, such as getting money, equipment and so on. I should like to mention this because the study of pulsars can serve as an example (not, of course, the only one) when the theorists have every reason for envying the observers. At any rate as far as I am concerned, this is so. In the previous report made by A. Hewish the facts were presented and we have every reason for congratulating the observers on their success. In less than three years great work has been done. As for the theory of pulsars we have, for the time being, a shortage of exactly established facts and I would like to discuss mainly general considerations and working hypotheses. Fortunately, for the theorists the situation is not always like that. There are cases when theory goes far ahead and anticipates observations. In the case of pulsars some lag in the theory is caused by two circumstances. Firstly we deal here with exceptionally complicated tasks, for example, with the equation of state of a substance with a density $\varrho \gtrsim 10^{11} \mathrm{~g} \mathrm{~cm}^{-3}$ and the electrodynamics of the magnetosphere of a rapidly rotating star with non-coinciding axes of rotation and of magnetic symmetry (say, with the direction of magnetic dipole). Secondly the observational data, in spite of their variety, give only indirect information about pulsars because their structure cannot be seen directly as, for example, in the case of the surface of the Sun or a number of nebulae.

All the foregoing, I believe, elucidates the character of the present report. It is devoted to the present situation of the theory of pulsars but we cannot yet present a clear and complete picture. 


\section{Physical Nature of Pulsars}

What are the pulsars as astronomical bodies? The main criterion in the choice of a candidate 'for pulsars' is the possibility to obtain a highly stable period which is rather small $\left(P=3 \times 10^{-2}-4 \mathrm{sec}\right)$. It is obvious enough that only a massive object can satisfy this requirement. So we have to deal with a star or a binary star but not with a nebula or a plasmatic bunch. Specifically, it has been suggested to identify pulsars with the following objects: (a) neutron stars, (b) white dwarfs, (c) double (binary) systems (binary stars), and (d) objects of a 'new type'.

From the very beginning it was suggested to regard pulsations [1] and rotation [2] as the mechanism providing the periodicity of the radiation pulses. At first the choice between these two possibilities was hampered because of the fact that only pulsars with periods $P \geqslant 0.25 \mathrm{sec}$ were known. After the discovery of the short periodic pulsars PSR 0833-45 and NP 0532 with periods 0.089 and $0.033 \mathrm{sec}$ the situation became much clearer*. The point is that because of the effects of general relativity the period of the fundamental mode of radial pulsations for non-rotating white dwarfs cannot be shorter than about $2 \mathrm{sec}$. For rotating white dwarfs the period of quasiradial pulsation can reach $0.6 \mathrm{sec}$ while the period of fundamental non-radial pulsation can reach $0.2 \mathrm{sec}$. And even if we disregard the difficulty of involving non-radial pulsations because of their damping owing to gravitational radiation, pulsation periods for white dwarfs $P<0.2 \mathrm{sec}$ can only be obtained for overtones. But how then, in accordance with the data on pulsars, can we explain the presence of pulsations in some overtone while it is completely absent for the fundamental tone and for other overtones? Besides, the question arises of the cause of the high stability of the pulsations.

Rotation periods of white dwarf are limited by the requirements that no collapse or intense outflow of matter from the star should occur. The latter condition can be observed, roughly speaking, the gravitational acceleration exceeds the centrifugal acceleration. Hence, we come to the inequality:

$$
\frac{G M}{r_{0}^{2}}>\frac{v_{0}^{2}}{r}=\Omega^{2} r_{0} \quad \text { or } \Omega<\left(\frac{G M}{r_{0}^{3}}\right)^{1 / 2}=\left(\frac{4 \pi G \bar{\varrho}}{3}\right)^{1 / 2},
$$

where $M$ is mass, $r_{0}$ the radius, $v_{0}=\Omega r_{0}$ is the velocity at the surface and $\bar{\varrho}$ is the average density of the star. Thus the rotation period should satisfy the condition

$$
P={ }_{\Omega}^{2 \pi}>\left(\frac{3 \pi}{G \bar{\varrho}}\right)^{1 / 2} \text {. }
$$

Hence $P>1 \mathrm{sec}$ when $\varrho \leqslant 10^{8} \mathrm{~g} \mathrm{~cm}^{-3}$. In the case of non-rigid rotation, the angular velocity, particularly near the poles can in principle be faster than according to the estimation (1). However, there are no indications of the possibility of obtaining values $P \leqslant 0.1 \mathrm{sec}$; practically, even rotation periods $P<1 \mathrm{sec}$ are hardly probable for

* The data on the pulsation periods and the rotation periods for white dwarfs and neutron stars are presented in detail in References [3-6] and in the literature cited there. 
white dwarfs. Thus it is almost sure that short periodic pulsars cannot be white dwarfs. Such a conclusion is also confirmed by the fact that not a single pulsar is identified optically with a white dwarf*. The parameters of neutron stars (their central density $\varrho_{c}$, radius $r_{0}$ and, for example, the period of the fundamental mode of radial pulsations $P_{0}$ ) depend on the equation of state of nuclear matter. Calculations whose results were used till recently for neutron stars showed their highest possible mass $M_{\max } \approx 1-2.5 M_{\odot}$; in this case for mass $M \approx M_{\odot}$ radius $r_{0} \approx 10 \mathrm{~km}$ and the period of pulsations $P_{0} \approx 10^{-3}-10^{-4} \mathrm{sec}$. According to these calculations [3] for light neutron stars $r_{0} \approx 50-200 \mathrm{~km}, \varrho_{c} \approx 3-10 \times 10^{13} \mathrm{~g} \mathrm{~cm}^{-3}$ and $P_{0} \approx 10^{-2} \mathrm{~s}$ at $M \approx 0.1-0.2 M_{\odot}$. On the other hand according to calculations [5] in which a more correct equation of state seems to be used $M_{\max } \approx 0.26 M_{\odot}$ at $\varrho_{c} \leqslant 10^{15} \mathrm{~g} \mathrm{~cm}^{-3}$ while at $\varrho_{c} \leqslant 3 \times 10^{14} \mathrm{~g} \mathrm{~cm}^{-3}$ $\left(M \lesssim 0.13 M_{\odot}\right)$ there are no stable configurations at all. An analogous assertion has been made in Reference [6] for the models with $\varrho \approx 10^{13} \mathrm{~g} \mathrm{~cm}^{-3}$.

It apparently results from this that for neutron stars $P<10^{-2} \mathrm{sec}$. Irrespective of possible future more precise calculations of pulsation periods for neutron stars, it is practically obvious that these periods are less than those for the observed pulsars. On the contrary, even the shortest known period $P=0.033 \mathrm{sec}$ is acceptable as a rotation period for neutron stars [7]. As a matter of fact, if $M \approx M_{\odot}$ and $r_{0} \approx 10^{6} \mathrm{~cm}$ we have an average density $\varrho \approx 5 \times 10^{14}$ and, according to (1) a rotation period $P>10^{-3}$ sec. Recent calculation [5], yield a radius of $r_{0} \approx 30 \mathrm{~km}$ for $M \approx 0.2 M_{\odot}$, i.e. $\bar{\varrho} \approx 4 \times 10^{12}$ and $P>10^{-2}$ sec.

So, on the basis of the observed periods all known pulsars can be rotating neutron stars. Pulsars with long periods $(P \gtrsim 1 \mathrm{sec}$ ) might be rotating or pulsating white dwarfs. But the latter assumption is unlikely in view of the absence of optical identifications of pulsars with white dwarfs, evolutional considerations (viz. the increase of the pulsars' period with time; due to this fact short periodic pulsars in the course of time have to become long periodic) and finally the absence of indications that there exist pulsars of various types.

The assumption that pulsars are members of binary systems (stars) seems to be altogether eliminated due to gravitational radiation. In view of such radiation the period $P \lesssim 1 \mathrm{sec}$ of a binary star should change much more rapidly and in the direction than is observed for pulsars (see e.g. the corresponding formula for $\mathrm{d} P / \mathrm{d} t$ in Reference [3]). In fact, some doubts have been expressed in the literature about the correctness of the assertion that binary stars are radiating gravitational waves. Regarding the theoretical aspect of the question these doubts, which have always seemed groundless to us (and to many others), are now strictly disproved.

We should like to mention also that gravitational radiation with a power of the same order of magnitude as in the general theory of relativity should result from any

\footnotetext{
* Pulsar NP 0532 in the Crab Nebula cannot be a white dwarf, because its optical emission between the pulses is practically absent. On the other hand white dwarfs, in principle, can be invisible (white dwarfs with a mass close to the critical limit cool down quickly), and for this very reason the fact that pulsars are not identified with white dwarfs cannot strictly prove the assumption that pulsars are not white dwarfs.
} 
other gravitational field theory which does not contradict with known experimental and observational data.

There remains to be discussed the supposition that pulsars are objects of a 'new type', for example something like tiny quasars (they could be called 'quasarino')*. More specifically, the question is whether the evolution or collapse of stars could lead to configurations different from white dwarfs, neutron stars and collapsed stars (in the latter case in a comoving system of reference the star can reach radii smaller than the gravitational radius $r_{g}=2 G M / c^{2} \approx 3 \times 10^{5} M / M_{\odot} \mathrm{cm}$ ). If we would not exceed the limits of the general theory of relativity, the only known possibility for searching for new dense quasistellar configurations is connected with considerations on the influence of a magnetic (or electromagnetic) field [8, 9]. We may believe, however, that the influence of a magnetic field can prove to be radical, provided the magnetic energy of the star is comparable with its gravitational energy, i.e. $W_{m} \approx$ $\approx\left(H^{2} / 8 \pi\right) r_{0}^{3} \approx G M^{2} / r_{0}$. Hence, if $M \approx M_{\odot}$, the field $H=10^{30} r_{0}^{-2}$, i.e., $H>10^{16}$ Oe at $r_{0}=10^{7} \mathrm{~cm}$. The appearance of such strong fields is hardly probable. It seems even less probable to identify pulsars with dense pulsating configurations which may be possible $[10,11]$ provided we refuse or at least modify the equations of general relativity. Some generalisation or modification of the equations of general relativity could, in principle, be expected, by taking into account the quantum fluctuations of the metrics which are significant for the characteristic length: $l_{g} \approx \sqrt{(}\left(G h / c^{3}\right)=10^{-33}$ $\mathrm{cm}$, time: $t_{g}=l_{g} / c=10^{-43} \mathrm{sec}$, and density: $\varrho_{g}=c^{5} / G^{2} \hbar=5 \times 10^{93} \mathrm{~g} \mathrm{~cm}^{-3}$. But the average density of a star with mass $M$ and radius $r \approx r_{g}=2 G M / c^{2}$ is about $\bar{\varrho}\left(r_{g}\right)=$ $=3 c^{2} /\left(8 \pi G r_{g}^{2}\right)$. It is obvious that for $M=M_{\odot}$, the density $\bar{\varrho}\left(r_{g}\right)=10^{16} \mathrm{~g} \mathrm{~cm}^{-3} \ll \varrho_{g}$ and $\bar{\varrho} \approx \varrho_{g}$ only for a 'star' with mass $M \approx M_{g} \approx \sqrt{ }(c h / G) \approx 10^{-5} \mathrm{~g}$, the gravitational radius of which is $r_{g} \approx l_{g} \approx 10^{-33} \mathrm{~cm}$. All this has nothing to do with pulsars. Hence, in order to make a pulsar model by changing the general theory of relativity these changes should be made at a rather 'early' stage, i.e. when gravitational fields are relatively weak.

There is no reason for that, but for those who would like to exceed the limits of already known physical laws and theories in astronomy, pulsars are one of the most attractive objects. We shall return to this at the end of this discourse. And now I should mention that from my judgement it is only accidentally that the identification of pulsars with neutron stars has not generally caused a storm of doubts** while the use of cosmological distances for quasars has been repeatedly disputed and is being questioned up to now. In any case we will make use of this lucky fact and will below consider pulsars to be rotating neutron stars.

\footnotetext{
* It is most probable that the quasar nucleus (compact source of powerful radiation) is a supermassive plasma body $\left(M \approx 10^{9} M_{\odot}, r \approx 10^{17} \mathrm{~cm}\right)$. Large internal rotational type motions and magnetic fields are characteristic of this body [8]. Therefore, some analogy between quasars and pulsars is evident (see also [8a]).

** The above-mentioned uncertainty which concerns the parameters of neutron stars $[3,5,6]$ does not excite any particular apprehension at present. For understanding the mechanism of the formation of neutron stars, the situation is worse but this process is so complicated that the difficulties that we have in mind [12] cannot yet arouse any fears.
} 


\section{Rotating Magnetized Neutron Stars}

In transforming the star into a neutron star the moment of inertia is considerably diminished (for example, if the mass does not change and the radius reduces from $3 \times 10^{10}$ to $3 \times 10^{6} \mathrm{~cm}$, the moment of inertia diminishes by 8 orders of magnitude). That is why it is quite natural to expect neutron stars to rotate rather rapidly (for NP 0532 the angular velocity $\Omega \approx 200$ as compared with that of the Sun's surface $\Omega_{\odot} \approx 2 \times 10^{-6}$ ).

As far as the magnetic field is concerned there is an analogous situation, i.e. in the case of 'frozen-in' magnetic field lines, the field $H$ increases proportionally to $r^{-2}$ or to $\varrho^{2 / 3}$ ( $r$ is some radius of the star, $\varrho$ is its density). Hence, for example, for a field $H \approx 1$ Oe at $r \approx 3 \times 10^{10} \mathrm{~cm}$ or $\varrho \approx 1 \mathrm{~g} \mathrm{~cm}^{-3}$ we obtain fields $H_{0} \approx 10^{8}$ at $r_{0} \approx 3 \times 10^{6}$ and $\varrho_{0} \approx 10^{12}$. The initial field of the star can, however, reach $10^{3}-10^{4} \mathrm{Oe}$ and central density of the neutron stars $\varrho_{c} \gtrsim 10^{14}-10^{15}$. That is why fields in neutron stars can (though they should not) reach values $10^{13}-10^{15}$ or, which is more realistic, values $H \approx 10^{12}$ Oe. On the other hand, due to the outflow of the envelope and for some other reasons the initial radius $r$ may be only about $10^{8} \mathrm{~cm}$. Then even at $r_{0} \approx 10^{6}$ and $H \approx 10^{4}$ the field $H_{0}=10^{8} \mathrm{Oe}$. (The possibility that magnetic fields of neutron stars can be so strong was noted even before the discovery of pulsars; see, for example $[8,13]$.

Thus we can say that neutron stars should, as a rule, rotate rather rapidly (angular velocity $\Omega \lesssim 10^{3}$ ) and should be highly magnetized (fields $10^{8} \lesssim H \lesssim 10^{12}-10^{14} \mathrm{Oe}$ ). There is no known reason, besides, for coincidence of the rotation axis $\mathbf{\Omega}$ with the magnetic moment $\mathbf{m}$ (or some other axis of magnetic symmetry) connected with the star. So we come to a non-symmetric and non-stationary system, i.e. to the model of the so-called oblique rotator (Figure 1). The discussion of such a model [14] in application to neutron stars had started not long before the discovery of pulsars.

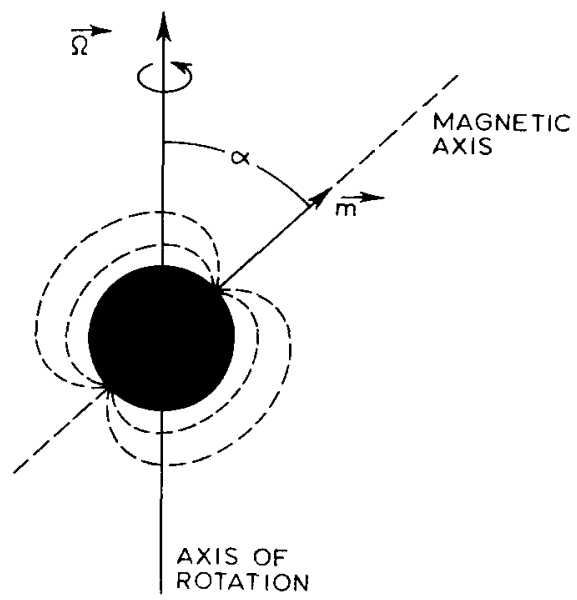

Fig. 1. Oblique rotator. 
There are two other circumstances in favour of the identification of pulsars with rotating neutron stars. First of all owing to the emission of electromagnetic and gravitational waves and also as a result of gas outflow (stellar wind) the angular velocity of the star should decrease. In accordance with this the period of pulsars $P$ should, as a rule, increase, which is being observed. Secondly, at any rate for the pulsar NP 0532 in the Crab Nebula the period of which doubles during a time interval $T \approx 2400 \mathrm{yr}$, it is natural to assume that the decrease of the kinetic energy of the stars' rotation $|\mathrm{d} K / \mathrm{d} t| \approx K / T=10^{-11} \mathrm{~K} \mathrm{erg} \mathrm{sec}{ }^{-1}$ is equal to the total luminosity of the Crab Nebula $L \approx 10^{38} \mathrm{erg} \mathrm{sec}^{-1}$. Such an assumption agrees well with the rough estimation of the kinetic energy of neutron star rotation $K=\frac{1}{2} I \Omega^{2} \approx 10^{49} \mathrm{erg}$, obtained for $\Omega=200$ and the moment of inertia $I \approx M r_{0}^{2}=10^{45} \mathrm{~g} \mathrm{~cm}^{2}\left(M \approx M_{\odot}=2 \times 10^{33} \mathrm{~g}\right.$, $r_{0}=10^{6} \mathrm{~cm}$ ).

Theoretical problems connected with the study of pulsars as rotating magnetized neutron stars are rather numerous but we can, though conditionally, outline three groups of questions:

Structure and processes within neutron stars.

Structure and dynamics of the atmosphere and magnetosphere of rotating neutron stars. Connection of the magnetosphere of the star with a supernova envelope and the interstellar medium.

Mechanisms of pulsar radiation and the corresponding models of pulsars - sources of observed electromagnetic radiation.

Besides, of course, several questions arise concerning the role of pulsars in connection with supernova remnants, their role as sources of cosmic rays, the application of pulsars to various astronomical and physical purposes and so on.

Below we are going to dwell briefly on all these aspects of the problem of pulsars.

\section{Pulsars and the Structure of Neutron Stars}

In view of the fact that we have not enough knowledge of the equation of state for the substance at superhigh densities, quantitative calculations of the structure of neutron stars are not yet reliable (see above and References $[5,6,15]$ ). We, however, will be interested below only in a qualitative picture and estimations of orders of magnitude. So it seems possible to choose the following parameters of a 'typical' neutron star $M=0.5 M_{\odot}, r_{0}=1-3 \times 10^{6} \mathrm{~cm}, \varrho_{c} \gtrsim 10^{15} \mathrm{~g} \mathrm{~cm}^{-3}$. Further, the density of the neutron liquid $\varrho_{n}$ is close to the total density of the substance in the star only at $\varrho \gtrsim 5 \times 10^{13} \mathrm{~g} \mathrm{~cm}^{-3}$. If $\varrho \$ 3 \times 10^{11}$ the role of neutrons is negligibly small and the substance consists of nuclei and electrons. Thus, it is quite clear that the outer layer of the star (say, at $\varrho \lesssim 10^{12}$ ) is a plasma and is similar to the substance in white dwarfs. But hence, a less evident assertion arises that the main part of the plasma layer of a neutron star is solid, i.e. forms a crust [15]. The point is that as a result of neutrino and electromagnetic radiation a neutron star cools rapidly and in view of the high heat conductivity it is rather soon after formation that practically the whole star has a temperature below $1-5 \times 10^{8} \mathrm{~K}$. At the same time the melting temperature $T_{m}$ of a 
plasma is determined from the condition $\Gamma k T_{m}=e^{2} Z^{2} / r_{i}$, where $r_{i}=n_{i}^{-1 / 3}$ is the average distance between nuclei with a charge $e Z$. The numerical factor $\Gamma=100-200$, i.e. the melting takes place when the kinetic energy of the nuclei is by two orders of magnitude less than the energy of their Coulomb interaction. Hence,

$$
T_{m} \approx 10^{3} \varrho^{1 / 3} Z^{5 / 3} \mathrm{~K}
$$

where the density $\varrho \approx 2 Z m_{p} n_{i}$, because we put $A / Z=2\left(m_{p}=1.67 \times 10^{-24} \mathrm{~g}\right.$ is the mass of the proton). It is clear from (2) that if $Z \gtrsim 10$ and $\varrho \gtrsim 10^{10}$ the temperature $T_{m} \gtrsim 10^{8}$. Thus except for a thin 'fluid' or a gaseous surface plasma layer, a rather considerable plasma part of the star should be solid. The thickness of this solid layer for a 'typical' neutron star is about $10^{4}-10^{5} \mathrm{~cm}$. Under the crust there is a neutron liquid $(\varrho>5-$ $10 \times 10^{13}$ ) with an additional concentration of protons and electrons of one or several per cent [16]. All these particles (neutrons, protons and electrons) form a degenerate Fermi system and under such conditions we can with a certain approximation assume that the system consists, in a sense, of a mixture of independent neutron, proton and electron Fermi liquids. The electron liquid of high density is always a normal Fermiliquid, i.e. it is close to a Fermi-gas. But neutron and proton liquids can undergo a corresponding transformation into a superfluid and superconducting state (see [17-22]).

It was only in 1957 that the nature of superfluidity and superconductivity in Fermisystems became clear (the theory of Bardeen, Cooper and Schriffer), though superconductivity of metals had been discovered about half a century earlier (in 1911). As it turned out, if in a degenerate Fermi gas (liquid) particles with energies close to the Fermi-energy attract each other they will stick together and form pairs even in the case of the weakest attraction. Being bosons these pairs undergo something analogue to the Bose-Einstein condensation. In other words, in the case of attraction the ordinary Fermi distribution proves to be unstable and the energy spectrum of the system shows a gap, the width of which $\Delta(T)$ depends on the temperature $T$; the gap is maximal and equal to $\Delta(0)$ at $T=0$. At a temperature $T_{c}$ called the critical temperature the gap is closed (i.e. $\Delta\left(T_{c}\right)=0$ ). The value of $T_{c} \approx \Delta(0) / K$ or, if we measure $\Delta(0)$ in $\mathrm{MeV} T_{c} \approx 10^{10} \cdot \Delta_{\mathrm{MeV}}(0) \mathrm{K}$.

The presence of an energy gap makes it impossible for the particles to scatter by collisions, and, therefore, their flow does not slow down, once it appears. Thus the system appears to be superfluid or in the case of charged particles it is superconducting. Neutrons with opposite spins (in the $s$-state) will attract each other if not too close. This attraction is not strong enough for the formation of a bineutron but if degeneracy takes place (i.e. for a rather dense neutron gas) this attraction should result in the formation of the pairs mentioned and in their condensation into a superfluid state. The maximum width of the gap $\Delta(0)$ is of the same order of magnitude or a bit less than the energy of nuclear interaction, i.e. about $1 \mathrm{MeV}$ and, therefore, the critical temperature

$$
T_{c} \approx 10^{10} \Delta_{\mathrm{MeV}}(0) \approx 10^{10} \mathrm{~K}
$$

This estimation refers to the density of a neutron liquid $\varrho_{n} \approx 10^{13}-10^{14} \mathrm{~g} \mathrm{~cm}^{-3}$ and, 
therefore, is suitable for the neutron liquid immediately under the crust (we shall recall that the total density of the substance $\varrho \approx \varrho_{n}$ at $\varrho \gtrsim 5 \times 10^{13}$, i.e. just in the inner border of the crust). With the growth of density, however, the considered gap $\Delta_{S}(0)$ for the pairs in the $S$-state decreases due to the increased role of repulsion and, according to some estimations $[21,22]$, the gap is 'closed', i.e. $\Delta_{S}(0)=0$ at $\varrho \approx \varrho_{n} \approx$ $\approx 1.5-2 \times 10^{14} \mathrm{~cm}^{-3}$ (density $\varrho_{n}=1.5 \times 10^{14} \mathrm{~g} \mathrm{~cm}^{-3}$ corresponds to the density of neutrons in atomic nuclei; the total density in nuclei is about twice as much, i.e. $\left.\varrho_{\text {nuicei }} \approx 3 \times 10^{14}\right)$. Nevertheless, superfluidity does not necessarily disappear at $\Delta_{S}(0)=0$, because at $\varrho \approx \varrho_{n}>1.5 \times 10^{14}$ the attraction between neutrons in the $p$ state (in the triplet state with spin 1) comes into effect. The corresponding gap $\Delta_{p}(0)$ is, apparently, a bit less than the gap $\Delta_{S}(0)$, but the rough estimation (3) remains valid [22].

A proton liquid behaves approximately like a neutron liquid but its density is less by one or two orders of magnitude. As a result of the Coulomb repulsion of protons, the corresponding gap is apparently less by an order of magnitude than for a neutron liquid, that is the critical temperature for proton superconductivity $T_{c} \approx 10^{9} \mathrm{~K}$.

Since in the majority of cases the temperature of the star $T \ll T_{c}$, we come to the

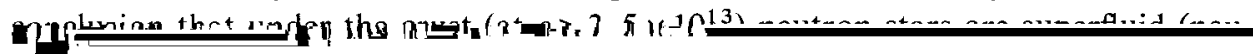

$\tau_{m}=4 \times 10^{6} \mathrm{yr}$ that may arise from the observed distribution of pulsar periods $[27$. (see, however, [27a, 47]), seems possible but it is far from being confirmed by th

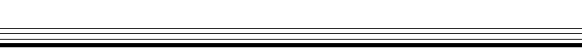
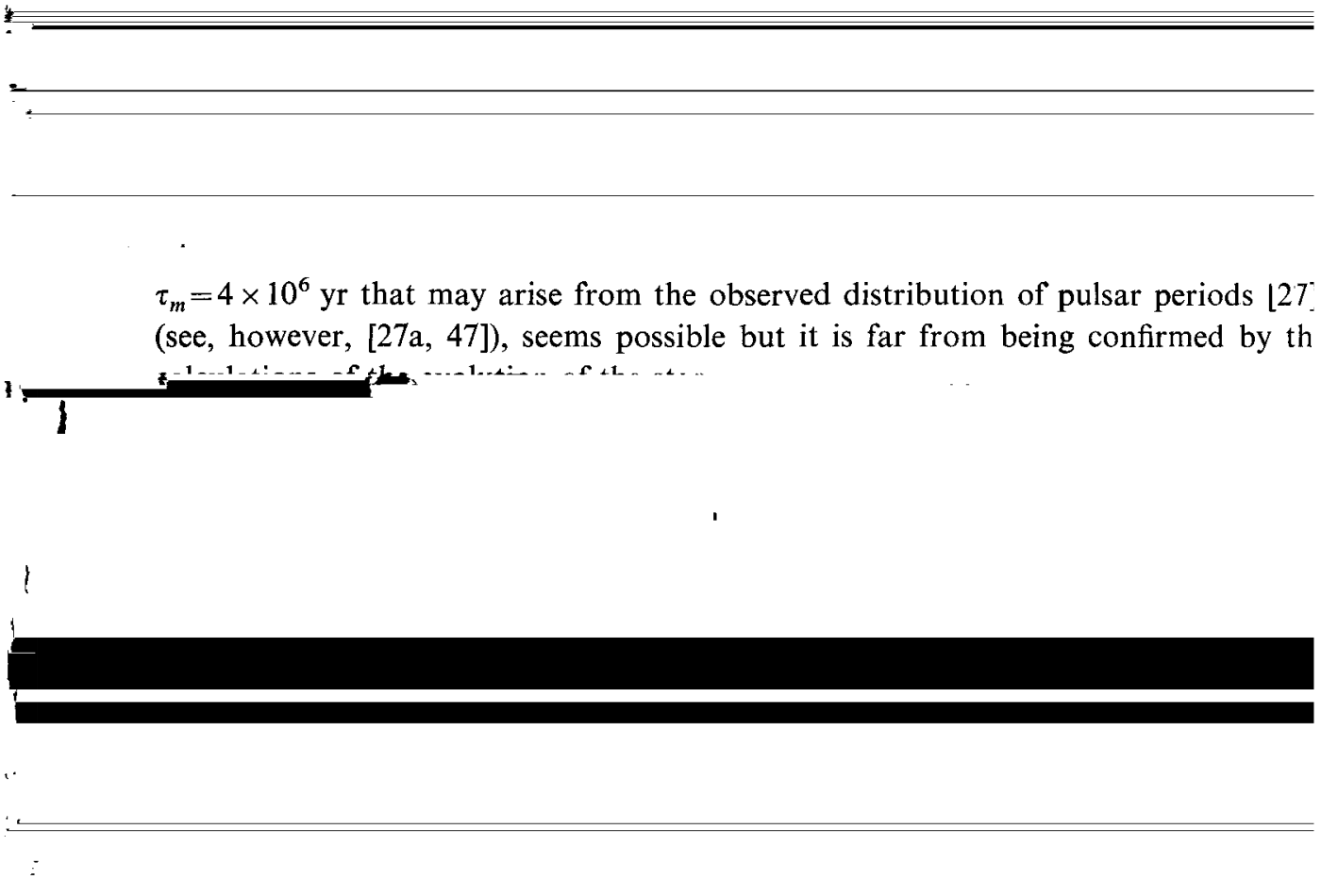
results in an increase of the succession period for pulsar pulses. But a dense crust cannot change its shape smoothly and it follows that with the rotation slowing down we should expect 'starquakes', that is the appearance of cracks and movements in the crust etc. As a result the crust's shape approximates the equilibrium shape for the given angular velocity.

There is every reason to believe that due to 'starquakes' and changes in the crust the angular momentum $J=I \Omega$ remains constant and, consequently, the change of the angular velocity is connected with the change of the moment of inertia $\Delta I$, so that $\Delta \Omega / \Omega=-\Delta I / I$. For PSR 0833-45 a change $\Delta \Omega / \Omega=2 \times 10^{-6}$ has been observed and, so $\Delta I \approx-2 \times 10^{-6} I \approx-10^{39} \mathrm{~g} \mathrm{~cm}^{2}\left(\Omega=70, I \approx M r^{2} \approx 10^{45} \mathrm{~g} \mathrm{~cm}^{2}\right.$ at $M \approx M_{\odot}$ and $r \approx 10^{6}$ ). Thus a mean radius of the star $r$ should decrease only by $\Delta r \approx|\Delta I| / I r \approx 1 \mathrm{~cm}(!)$. It seems that after a change in the crust the star's rotation should be the same as before the crust's rebuilding because a negligibly small change in the radius is unlikely to influence the breaking torque (see, however, below). Actually, after the 'catastrophe' the pulsar in Vela began to slow down quicker than before the 'catastrophe' (specifically, $\Delta \dot{\Omega} / \dot{\Omega} \approx 10^{-2}, \dot{\Omega} \equiv \mathrm{d} \Omega / \mathrm{d} t$ ). A rather plausible explanation [30] of this effect associates it with superfluidity and superconductivity of the neutronproton liquid under the crust.

In superfluid liquids* with temperatures much less than the critical value, only motions without vorticity can take place and a superfluid part of the star does not seem to be able to rotate, that is its effective moment of inertia is to be equal to zero. Practically, however, already at negligibly small angular velocity $\Omega_{c} \approx\left(\hbar / m_{n} r^{2}\right) \ln (r / a) \approx$ $\approx 10^{-4} \sec ^{-1}\left(m_{n} \approx 10^{-24}\right.$ is the mass of a nucleon, $r$ is the radius of a fluid sphere and $a=10^{-12} \mathrm{~cm}$ is the radius of the core of a vortex line where the liquid is not yet a superfluid) the appearance of vortex lines in the rotating superfluid parallel to the rotation axis is energetically advantageous. Round every such line the velocity circulation is equal to $2 \pi h / 2 m_{n}$ and the angular momentum of the pair of nucleons (with mass $2 m_{n}$ ) as a result of their motion around the line is equal to $\hbar=1.05 \times 10^{-27}$ erg sec. If $\Omega \gg \Omega_{c}$ the number of lines formed is rather large for the angular velocity of the substance to be the same as it is in the case of a normal liquid. Then the number of vortex lines per unit area perpendicular to the rotation axis $n_{0}=2 m_{n} \Omega / \pi \hbar$ (star's angular momentum

$$
J=I \Omega \approx M r^{2} \Omega \approx n_{0} \hbar \pi r^{2} \stackrel{M}{2 m_{n}},
$$

where $M / 2 m_{n}$ is the total number of pairs). Hence, the mean distance between the vortex lines is $\xi=n_{0}^{-1 / 2} \approx\left(\hbar / m_{n} \Omega\right)^{1 / 2} \approx\left(10^{-3} / \Omega\right)^{1 / 2}$. Even for the pulsar in the Crab Nebula with $\Omega \approx 200$ the distance $\xi \approx 2 \times 10^{-3} \mathrm{~cm}$, which is incomparably larger than the mean distance between the neutrons $\xi_{n} \approx\left(\varrho / m_{n}\right)^{-1 / 3} \approx 10^{-13} \mathrm{~cm}$.

* On the Earth the superfluidity has been investigated and is known only for helium II (liquid helium at $T<T_{\lambda}=2.17 \mathrm{~K}$; we do not touch upon solutions of $\mathrm{He}^{3}$ in $\mathrm{He}^{4}$ ). In this case the superfluidity disappears at the $\lambda$-point (at a temperature $T_{\lambda}$ when helium II transforms into helium I), corresponding to the temperature of the Bose-Einstein condensation of helium atoms. 
Thus in a rotating neutron star its neutron-proton (fluid) part participates in rotation due to the appearance of a set of vortex lines which are probably somehow anchored to the solid crust. It the rotation velocity does not change the presence of vertical lines inside will not of course pronounce itself outside. But if the angular velocity changes, the situation is different. In the case of a normal (non-superfluid) liquid neutrons can exchange momentum with protons and electrons fairly rapidly (characteristic time $\tau \approx 10^{-15} \mathrm{sec}$; see [20]). If the protons are superconducting and the neutrons are normal the momentum is transferred due to the interaction of electrons with the magnetic moment of neutrons and $\tau \approx 10^{-9} \mathrm{sec}$. If protons are superconductive and the neutrons form a superfluid, momentum transfer takes place only in the normal cores of vortex lines. The volume of these cores is by a factor

$$
\pi a^{2} n_{0} \approx \pi a^{2} \frac{2 m_{n} \Omega}{\pi \hbar} \lesssim 10^{-18}
$$

less than the total volume of a neutron liquid. In view of this the time of relaxation $\tau$ becomes days or years (!). Hence, with the decrease of the moment of inertia of the crust only its angular velocity and the angular velocity of the proton and electron liquids increases at the beginning of the process while the angular velocity of the superfluid neutron layer will undergo some changes during a time $\tau_{0}=0.1-10$ years. Thus the star's rotation can be described, in first approximation, by the equations [30] that speak for themselves

$$
\begin{gathered}
I_{c} \dot{S}=\mathscr{N}-\frac{I_{c}}{\tau_{0}}\left(\Omega-\Omega_{n}\right) \\
I_{n} \dot{Q}_{n}=I_{c} / \tau_{0}\left(\Omega-\Omega_{n}\right) .
\end{gathered}
$$

Here $I_{c}$ and $I_{n}$ are the moments of inertia for the crust and the superfluid part of the star respectively, $\mathscr{N}$ is the breaking torque, $\Omega$ is the observed angular velocity of the crust and $\Omega_{n}$ is the angular velocity of the superfluid liquid. We can not spend time on the analysis of the Equations (5), specifically, under the change of the moments of inertia $I_{c}$ and $I_{n}$ resulting from 'starquakes'. Qualitatively, however, it is quite clear that before the establishment of a quasi-equilibrium (i.e. for a time $t \lesssim \tau_{0}=0.1-10$ yr) the crust is decelerated more strongly than the neutron liquid. So it is natural that after a 'starquake' (and, by the way, independent of the nature of the jump of angular velocity) the pulsar's period increases more rapidly than before. It results from this theory that $\Delta \dot{\Omega} / \dot{\Omega} \approx\left(T / \tau_{0}\right) \cdot(\Delta \Omega / \Omega)$, where $T$ is a characteristic time for the star's rotation slowing down, for example, the time of doubling of the pulsar period (we may as well assume that $Q / \Omega=-1 / T$ ). For the pulsar in Vela $T=24000 \mathrm{yr}$ and for $\tau_{0}=1$ yr $\Delta \dot{Q} / \dot{\Omega} \approx 10^{4} \Delta \Omega / \Omega$, which corresponds to the observations.

The non-monotonous change of the frequency ('frequency wobble') with a period of about three months registered for the pulsar NP 0532 can also be connected with the behaviour of a superfluid part of the star [32]. The point is that slow torsional oscillations (just with the needed period) can take place in the system of vortex lines.

It should be noted that the discovered perturbations in the monotonous increase 
of the periods of the young pulsars in Vela and Crab may, in principle, not only be associated with 'starquakes' of the crust and with superfluidity of the neutron liquid. We might try to connect the perturbations of the period with the presence of light satellites (planets) [33,34] of a pulsar-star, with changes of gravitational radiation losses due to changes of quadrupole moment of the star's mass [34a] and with some other reasons $[33,34]$ * However, the above explanation seems to us more probable; it can be tested during sufficiently long series of observations over the change of the pulsar period after the 'catastrophe' (the jump of the period).

Thus, the study of irregularities (perturbations) in the course of a secular increase of the pulsar period offers the possibility to 'look' inside a neutron star. Preliminary data on this account testify in favour of the presence in neutron stars of a solid crust and a superfluid neutron core or layer (the protons in this layer are, probably, superconducting).

We cannot but emphasize that after the failure of the efforts to identify some cosmic X-ray sources with neutron stars and up to the discovery of pulsars the prospect of proving the very existence of neutron stars did not seem cheerful. Now we may even study the inner parts of these stars and we feel inspired by this success.

\section{Electrodynamics of Rotating Magnetized Neutron Stars}

The above estimates of the time of magnetic field relaxation $\tau_{m}$ allow us in first approximation to consider that in a coordinate system connected with the star its magnetic field does not change with time (within the star's body itself). The structure of this field is not known and there is no reason to consider it to be strictly dipolar but generally a dipole term is of primary importance outside the star. Nevertheless, strictly speaking, this refers only to the case when a star is in vacuum. If in the magnetosphere of the star or even in its wave zone a rather dense plasma is present the character of the field outside the star can change drastically.

Let us assume first that the star is in vacuum. In this case a correct solution is known for the field of a rotating star as far as it can be considered to be a point magnetic dipole and for a more realistic model of a perfectly conducting, uniformly magnetized rotating sphere [35]. The magnetic dipole moment of the star $\mathbf{m}$ can conveniently be split into a component $\mathbf{m}_{\|}$along the rotation axis $\mathbf{\Omega}$ and a component $\mathbf{m}_{\perp}$ perpendicular to it.

Obviously, $\mathbf{m}_{\|}$does not change with time (we assume here that $m=$ const), while the dipole $\mathbf{m}_{\perp}$ rotates and, therefore, radiates. Irrespective of details of the structure of the field at the star's surface and near the star (nearby zone), in the wave zone (at $r \gg \lambda_{0}=2 \pi c / \Omega$ ) the field decreases as $1 / r$ and the total intensity (luminosity) of the

* We should note that, in using the Equations (5) in paper [30] and in all others known to us, the torque $\mathscr{N}$ is considered the same before and after the starquake. Meanwhile, not only gravitational braking (see [34a]) but, in all probability, a more sufficient electromagnetic braking for pulsars can be changed during a starquake as a result of the additional outflow of plasma and caused by the change of the conditions near the star (see the next part of this article). 
magnetic dipole radiation $L_{m}=\frac{2}{3} m_{\perp}^{2} \Omega_{c^{3}}^{4}$. It is natural that this intensity is gained due to the decrease of the kinetic energy of the star's rotation $K=I \Omega^{2} / 2$ and, therefore, at the absence of other losses:

$$
\begin{aligned}
& \mathrm{d} K \\
& \mathrm{~d} t
\end{aligned}=I \Omega \dot{\Omega}=-\frac{2}{3} m_{\perp}^{2} \Omega^{4} .
$$

Hence $\Omega=\Omega_{0}\left(1+t / T_{m}\right)^{-1 / 2}, T_{m}=3 c^{3} I / 4 m_{\perp}^{2} \Omega_{0}^{2}$ and the time $t$ is counted from the moment when $\Omega=\Omega_{0}$. In our epoch the period of the pulsar NP 0532 in the Crab Nebula doubles in a time $T \approx 2400 \mathrm{yr}=10^{11} \mathrm{sec}$. Hence, if $\Omega_{0} \approx 200$ and $I \approx M r_{0}^{2} \approx 10^{45}$ $\mathrm{g} \mathrm{cm}^{2}$ the time $T=T_{m}$ at $m_{\perp} \approx 2 \times 10^{30} \mathrm{G} \mathrm{cm}^{3}$. The field of the magnetic dipole $\mathbf{B} \equiv$ $\equiv \mathbf{H}=2 \mathbf{m} / r^{3}$ at the magnetic pole and $\mathbf{H}=-\mathbf{m} / r^{3}$ at the magnetic equator. Hence, if $m \approx m_{\perp} \approx 10^{30}$ at the surface of a star with a radius $r_{0} \approx 10^{6} \mathrm{~cm}$ the field $H_{0} \approx 10^{12}$ Oe. Such and estimate is usually used [36]. It does not contradict the independent estimations of the field connected with considerations of the formation of a neutron star (see above). At the same time it is obvious that Equation (6) is quite true only for a star in vacuum (we include the assumption that there is no particle outflow from the star) and if also the gravitational radiation is neglected. The role of gravitational radiation can be included by adding to the right side of Equation (6) the term

$$
-L_{g}=-\frac{G}{45} \mathscr{D}_{\perp}^{2} \frac{\Omega^{6}}{c^{5}} \approx-\frac{6 G}{c^{5}} I^{2} \varepsilon \Omega^{6},
$$

where $\mathscr{D}_{\perp}$ is the component of the quadrupole moment of the star's mass perpendicular to the rotation axis and $\varepsilon \approx(a-b) / a$ its ellipticity ( $a$ and $b$ are the axes of the star's elliptic cross-section perpendicular to the rotation axis; see [36]). In the oblique rotator model the moment $\mathscr{D}_{\perp}$ appears automatically under the influence of a magnetic field, asymmetric to the axis. However, only in the presence of an inner (for instance, toroidal) field in the star $H_{i} \gtrsim 10^{15}$ (see $[36,37]$ ) the intensity of gravitational radiation $L_{g} \approx 10^{38} \mathrm{erg} \mathrm{sec}^{-1}$. Though the presence of such a field is in principle acceptable and does not contradict the presence of an outer (for instance, poloidal) field $H_{0} \lesssim 10^{13}$, it seems to us rather unrealistic. For some reasons (see below) it is hardly possible also to estimate the intensity of gravitational radiation, considering either the energy balance or the dependence of the pulsar period (angular velocity) on the time. So, we can make things clear only by measuring the flux of gravitational waves emitted from the Crab pulsar (it is of importance that the plasma envelope practically does not affect the intensity of gravitational radiation). Unfortunately, such measurements are unlikely to be carried out in the nearest future*.

* The flux on the Earth

$$
t_{g} \equiv F_{g} \approx \underset{4 \pi \Re^{2}}{L_{g}} \approx 3 \times 10^{-7} \mathrm{erg} / \mathrm{cm}^{2} \mathrm{sec},
$$

corresponds to a gravitational radiation of the pulsar in the Crab Nebula with a power $L_{g} \approx 10^{38} \mathrm{erg} \mathrm{sec}^{-1}$ while the existing receivers can record only a flux $F_{g} \approx 10^{4} \mathrm{erg} \mathrm{cm}^{-2} \mathrm{sec}^{-1}$. Excluding any new methods [39] the recording of fluxes $F_{g}<10^{-6}-10^{-7} \mathrm{erg} \mathrm{cm}^{-2} \mathrm{sec}^{-1}$ will require the cooling of a receiver weighing several tons to very low temperatures $\left(T \lesssim 10^{-2} \mathrm{~K}\right)$. Such a project seems quite possible even today [40] but it will require rather hard work. 
Besides by electromagnetic and gravitational radiation, a star can lose its energy by the outflow of the star's plasma and the acceleration of charged particles escaping from the star [41-43]. It is of importance that near a magnetized star rotating in vacuum an electric field $E \approx(\Omega r / c) H$ should be present (in a coordinate system rotating with the star $E=0$ ).

Such an effect (as a matter of fact, we speak of unipolar induction) can play a significant part even in the case of ordinary slowly rotating stars [44]. Its role for pulsars, of course, increases considerably due to the high values of $H$ and $\Omega$. For example, if $\Omega \approx 10^{2}, r \approx 10^{6}$ and $H \gtrsim 10^{8}$ the field $E \gtrsim 3 \times 10^{5} \approx 10^{8} \mathrm{~V} / \mathrm{cm}$. Hence, we have a potential difference $V \approx E r \approx 10^{14} \mathrm{~V}$. It is quite obvious that in the presence of such fields and even with fields, smaller by several orders of magnitude (a field can considerably decrease if the plasma atmosphere of the star is dense enough) the gravity in the atmosphere of the star is of secondary importance. Specifically, it is out of the question that a magnetized rotating neutron star could have an extremely thin equilibrium atmosphere with a characteristic height $h \approx \kappa \operatorname{Tr}^{2} / G M m_{p} \approx \mathrm{I} \mathrm{cm}$ (at $T=10^{6} \mathrm{~K}, M=M_{\odot}, r_{0}=10^{6} \mathrm{~cm} ; m_{p}=1.67 \times 10^{-24}$ is the mass of a proton). Thus there is no reason and, generally speaking, it is impossible to consider such a star to be situated in a vacuum.

Unfortunately, it is extremely difficult to make a somewhat consistent theory of the atmosphere (magnetosphere) of a rotating magnetized neutron star which is already clear from the example of slowly rotating stars [45]. Possibly, the influence of the effects of general relativity may be significant for neutron stars and for the determination of electromagnetic fields $[8,46]$. In any case, no such theory has been made and the picture is obscure, even quantitatively if we speak of the distribution of plasma outside the star depending on the parameters $\Omega$, $\mathbf{m}$ (magnetic moment) and $\mathbf{r}$ (coordinates)*.

That is why we can make below only some remarks concerning the electrodynamics of pulsars.

Corotation of a star and plasma in its magnetosphere is altogether impossible for distances (from the rotation axis) exceeding the radius of the 'light cylinder'

$$
r_{c}={ }_{\Omega}^{c}=4.8 \times 10^{9} P(\mathrm{sec}) \mathrm{cm} .
$$

The point is that in the case of corotation (rigid rotation) even at $r=r_{c}$ the plasma velocity $v=c$. If $\Omega=2 \pi / P=200$ the radius $r_{c}=1.5 \times 10^{8} \mathrm{~cm}$ and at $H_{0} \gtrsim 10^{8}$ the field $H_{c} \approx H_{0}\left(r_{0} / r_{c}\right)^{3} \gtrsim 10^{2}$ Oe. Hence, $H^{2} / 8 \pi \approx n \kappa T$ for the number density of nonrelativistic electrons in the magnetosphere $n=n_{e} \gtrsim 400 / k T \approx 4 \times 10^{12} \mathrm{~cm}^{-3}$ at $T \sim 10^{6} \mathrm{~K}$ or for relativistic electrons $n=n_{r} \gtrsim 4 \times 10^{7} \mathrm{~cm}^{-3}$ at $\kappa T \approx E \approx 10^{-5} \approx 10 \mathrm{MeV}$. Hence,

\footnotetext{
* The dependence of the plasma density on the mass of the star and its temperature is, probably, of less significance. The same refers to the conditions far from the star if accretion does not play a role (according to [47] accretion may be of great importance). We should also note that the parameter $\mathbf{m}$ is equivalent to two scalar parameters, that is the field $H_{0}$ at the surface of the star (say, at its magnetic pole) and the angle $\alpha$ between $\Omega$ and $\mathbf{m}$.
} 
it is clear that as far as short periodic pulsars are concerned their magnetic field can really carry along a fairly dense plasma till distances $r \approx r_{c}$. However, even in this case, without speaking of long periodic pulsars, the rigid corotation of plasma can probably brake much earlier, at $r<r_{c}$ or even at $r \ll r_{c}$ depending on a number of circumstances, like the density and effective temperature of the plasma, the configuration of the field. Thus the distance $r_{c}$ plays the role of some maximum characteristic distance for pulsars. This conclusion corresponds with the estimate of the maximum length $l$ of the pulsar radiating region. In fact, pulses with a duration $\delta P$, generally speaking, should emerge from a region with a length $l \leqslant c \delta P$, because otherwise the pulse will be considerably blurred due to the dealy of the signals emerging from different parts of the source*. For pulsars, of course, the duration of the pulses $\delta P<P$ and, therefore, $l<c P=2 \pi c / \Omega$ or, practically, $l \ll 2 \pi c / \Omega$.

For the oblique rotator (angle $\alpha>0$ ) the picture is nonsymmetric and nonstationary. So there is every reason for expecting the appearance of different plasma instabilities causing plasma turbulence, its warming and further acceleration of particles in the magnetosphere and when leaving it. All these processes can, however, take place also in the case of coincidence of the rotation axis with that of magnetic dipole (angle $\alpha=0$ ). Such a model lends itself easier to the analysis [42] because of the presence of axial symmetry and, protably, reflects some significant peculiarities of more real pulsar models. It is interesting that for the model with axial symmetry, the slowing down of the star's rotation, though not taking place because of the radiation but due to the acceleration of particles by the electric field, corresponds to the formula (6) in which $m_{\perp}$ is replaced by $m_{\| \mid}$(see [41, 42]; of course, if $\alpha=0$ the magnetic moment along the rotation axis $\mathbf{m}_{\|}$is equal to the total moment $\mathbf{m}$ ). As a result, for the field $H_{0}$ at the star's surface we have the same estimate as before, i.e. for the pulsar in Crab $H_{0} \approx 10^{12}$ Oe.

However, such an evaluation of the field seems to us not yet convincing because the influence of plasma outside the star's body has not been included.

Most clearly it can be seen in the case of braking due to magnetic dipole radiation. As was noted above a magnetic dipole in vacuum radiates electromagnetic waves with the power

$$
L_{m}=\frac{2}{3} m_{\perp}^{2} \Omega^{4} c^{3}
$$

If, however, such a dipole is placed in a uniform isotropic medium with the index of refraction $\tilde{n}$, the power $L_{m}(\tilde{n})$ changes by a factor $\tilde{n}^{3}(\Omega)$ (for an electric dipole the power changes by a factor $\tilde{n}(\Omega)$ ). More precisely, the above concerns the case when waves with a frequency $\Omega$ can propagate in the given medium. If $\tilde{n}^{2}(\Omega)<0$ the waves do not leave the dipole and one obtains for the power of radiation $L_{m}(\tilde{n})=0$. For an

* More precisely, not the whole length of the radiating source appears in the role of $l$ but only the length of that part in which the intensity of radiation increases considerably due to the maser effect (for details see [48]). 
isotropic 'cold' (non-relativistic) plasma

$$
\tilde{n}^{2}(\omega)=1-\frac{\omega_{e}^{2}}{\omega^{2}}, \quad \omega_{e}=\sqrt{\frac{4 \pi e^{2} n_{e}}{m}}=5.64 \times 10^{4} \sqrt{n_{e}}
$$

and the inequality $\tilde{n}^{2}(\omega \equiv \Omega)<0$ can be fulfilled easily.

In the case of magnetic stars and specifically of pulsars a near-stellar plasma is influenced by the magnetic field. That means that the plasma is magnetoactive and the indices of refraction $\tilde{n}^{2}$ for normal waves propagating in it depend in a rather complicated way on the frequency $\omega$, the value of the field $H$, the angle $\theta$ between $\mathbf{H}$ and the wave vector $\mathbf{k}$ and on other parameters (see, for example, [49]). Thus, if the frequency $\omega$ is small as compared with the gyrofrequency for ions $\Omega_{H}=e H / m_{i} c=$ $=9.6 \times 10^{3} H$ (the ions are considered to be nonrelativistic; the numerical coefficient refers to the case of hydrogen when $m_{i}=m_{p}=1836 \mathrm{~m}=1.67 \times 10^{-24} \mathrm{~g}$ ), in a great number of cases a magnetohydrodynamic approximation is applicable. With this, for example, for waves propagating along the magnetic field

$$
\tilde{n}^{2}=1+\frac{4 \pi m_{i} n_{i} c^{2}}{H^{2}} \approx 1+\frac{4 \pi \varrho c^{2}}{H^{2}}
$$

or

$$
\tilde{n}=\frac{c \sqrt{4 \pi \varrho}}{H}=c / v_{a}
$$

on the condition that the Alfvén velocity

$$
v_{a}=\frac{H}{\sqrt{4 \pi \varrho}} \ll c .
$$

On the same condition, obviously, $\tilde{n} \gg 1$ (for example, if $H=10^{6}$, the particle density $n_{i}=n_{e}>10^{14} \mathrm{~cm}^{-3}$, and $\varrho>10^{-10} \mathrm{~g} \mathrm{~cm}^{-3}$, the index $\tilde{n}>10$ ). Thus for the magnetohydrodynamic region of frequencies the radiation and, therefore, the slowing down of the oblique rotator can sufficiently differ from that in vacuum (this conclusion is confirmed by more detailed calculations [50]). In the Crab Nebula far from the pulsar $H \lesssim 10^{-3}$, the ionic gyrofrequency $\Omega_{H} \lesssim 10$, and the magnetohydrodynamic approximation is not valid. On the contrary, in this case the influence of ions is usually negligible. Besides, it is most probable that in the Crab Nebula the plasma frequency for electrons $\omega_{e}=5.64 \times 10^{4} \sqrt{ } n_{e} \gg \omega_{H}=e H / m c=1.76 \times 10^{7} H$ and $\omega_{e} \gg \Omega \equiv \omega$. The same conditions are realized for whistlers in the Earth's magnetosphere and for 'spiral waves' in metals. As we know (see, for example, [49] § 11) waves of only one type (the ordinary wave) can propagate in the conditions mentioned, it being known that

$$
\begin{aligned}
& \tilde{n}_{2}(\Omega)=\frac{\omega_{e}}{\sqrt{\left(\Omega \omega_{H} \cos \theta\right)}}=\sqrt{\frac{4 \pi|e| n_{e} c}{\Omega H \cos \theta}}, \\
& \omega_{e} \gg \omega_{H}, \quad \omega_{e} \gg \Omega, \quad \omega_{H} \cos \theta \gg \Omega .
\end{aligned}
$$


If $\cos \theta \approx 1, \omega_{e} \approx 10^{5}-10^{6}\left(n_{e} \approx 1-10^{3}\right), \Omega=200$ and $\omega_{H} \approx 10^{4}\left(H \approx 10^{-3}\right)$, the index $\tilde{n}^{2}(\Omega) \approx 10^{2}-10^{3}$. If we assume just for orientation purposes that the radiation of the magnetic dipole is proportional to $\tilde{n}^{3}=\tilde{n}_{2}^{3}$ as well as in the isotropic medium, the losses in the discussed case would increase by $10^{6}-10^{9}$ times. With this the estimation of the field $H_{0}$ at the star surface would be lower by a factor $\tilde{n}_{2}^{3 / 2} \approx 10^{3}-3 \times 10^{4}$ as compared with the evaluation for the vacuum.

The above reasoning is not at all sufficient for a realistic estimation of the influence of the plasma on the slowing down of the star's rotation but nevertheless it illustrates the possibility for a nearstellar plasma to change radically all the picture and to cause the observed slowing down of the pulsar for fields $H_{0} \approx 10^{8}-10^{9} \mathrm{Oe}$ at the surface of the star. This is the reason why we believe the question of the magnitude of this field still to be open and the values which are often used $H_{0}=10^{12}-10^{13} \mathrm{Oe}$ can by no means be considered as well grounded.

Electromagnetic radiation does not only slow down the star's rotation but it may lead also to a change of the angle $\alpha$ between the axis of rotation $\boldsymbol{\Omega}$ and the magnetic moment $\mathbf{m}$. This question was discussed in articles [36, 51, 52] by determining the torque $\mathbf{N}$, which can be expressed through a stress tensor of the electromagnetic field $T_{i j}$. For the same purpose we may use the general equation for a 'particle' with a mechanical angular momentum $\mathbf{J}_{0}$ and magnetic moment $\mathbf{m}$

$$
\frac{\mathrm{d} \mathbf{J}_{0}}{\mathrm{~d} t}=\left[\mathbf{m} \mathbf{H}_{\mathrm{ext}}\right]-\frac{4 v_{m}}{3 \pi c^{3}}\left[\mathbf{m} \frac{\mathrm{d}^{2} \mathbf{m}}{\mathrm{d} t^{2}}\right]+\frac{2}{3 c^{3}}\left[\mathbf{m} \frac{\mathrm{d}^{3} \mathbf{m}}{\mathrm{d} t^{3}}\right],
$$

where $\mathbf{H}_{\text {ext }}$ is the outer magnetic field in the location of the dipole and $v_{m} \approx c / r_{0}$ depends on the structure (form-factor) of a dipole ( $r_{0}$ is the radius of the magnetized sphere)*. The torque

$$
\left.\mathbf{N}_{c}=-4 v_{m}\left[\begin{array}{c}
\mathrm{d}^{2} \mathbf{m} \\
\mathbf{m} c^{3}
\end{array}\right]=-\begin{array}{c}
\mathrm{d} \mathbf{J}_{m} \\
\mathrm{~d} t^{2}
\end{array}\right]
$$

* The torque affecting the sphere with magnetization

is equal to

$$
\mathbf{M}=\mathbf{m} \mathscr{D}(\mathbf{r}), \int \mathscr{D}(\mathbf{r}) \mathrm{d} \mathbf{r}=1
$$

$$
\int[\mathrm{mH}(\mathbf{r})] \mathscr{D}(\mathbf{r}) \mathrm{d} \mathbf{r},
$$

while the field $\mathbf{H}(\mathbf{r})=\mathbf{H}_{\text {ext }}+\mathbf{H}_{1}(\mathbf{r})$ where $\mathbf{H}_{1}(\mathbf{r})$ is the self-field of the dipole (sphere) at the point $\mathbf{r}$ (we consider the field $\mathbf{H}_{\text {ext }}$ to be uniform within the limits of the sphere). If we exclude the field $\mathbf{H}_{1}$ with the help of the field equations we come [53] to Equation (9). Both this equation and the way of obtaining it are analogous to what takes place when we consider the radiation friction force in the case of a charge (we speak of the equation

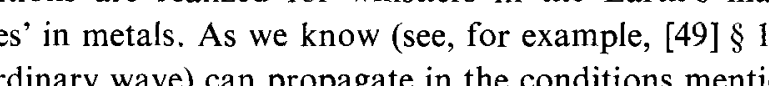

where $m_{0}$ is a mechanical mass,

$$
m_{\mathrm{em}}=\underset{c^{3}}{e^{2} v_{m}} \approx c^{2} r_{0}^{2}
$$

is the electromagnetic mass of a 'particle' the charge density of which

$$
\left.\varrho=e \mathscr{D}(\mathbf{r}), \int \mathscr{D}(\mathbf{r}) \mathrm{d} \mathbf{r}=1\right) .
$$


is conservative while

$$
\mathbf{J}_{m}=\frac{4 v_{m}}{3 \pi c^{3}}\left[\mathbf{m} \frac{\mathrm{d} \mathbf{m}}{\mathrm{d} t}\right]
$$

is the electromagnetic angular momentum of the star. If the outer field is absent $\left(\mathbf{H}_{\mathrm{ext}}=\mathbf{0}\right)$ and the dissipation is neglected the total momentum $\mathbf{J}=\mathbf{J}_{0}+\mathbf{J}_{m}$ is, of course, constant. For neutron stars $\mathbf{J}_{0}=I \Omega \lesssim 10^{47} \mathrm{~g} \mathrm{~cm}^{2} \sec ^{-1}$ (at $I \lesssim 10^{45}$ and $\Omega \lesssim 10^{2}$ ) and $J_{m} \approx m^{2} \Omega / r_{0} c^{2} \lesssim 10^{35} \mathrm{~g} \mathrm{~cm}^{2} \mathrm{sec}^{-1}$ (at $r_{0} \gtrsim 10^{6}, m \approx H_{0} r^{3} \lesssim 10^{30}$ ).

The moment $\mathbf{J}_{m}$ as well as any analogous quantity in which account is taken of the plasma influence is probably of interest in the case of a more detailed analysis of the star's dynamics. But for the decrease of the angular velocity $\Omega$ and the change of the angle $\alpha$ it is the dissipative torque that is of importance

$$
\begin{aligned}
\mathbf{N}_{d} & =\frac{2}{3 c^{3}}\left[\mathbf{m} \frac{\mathrm{d}^{3} \mathbf{m}}{\mathrm{d} t^{3}}\right]=\mathbf{N}_{d \|}+\mathbf{N}_{d \perp} \\
\mathbf{N}_{d \|} & =\frac{2}{3 c^{3}}\left[\mathbf{m}_{\perp} \frac{\mathrm{d}^{3} m_{\perp}}{\mathrm{d} t^{3}}\right], \quad \mathbf{N}_{d \perp}=\frac{2}{3 c^{3}}\left[\mathbf{m}_{\|} \frac{\mathrm{d}^{3} \mathbf{m}_{\perp}}{\mathrm{d} t^{3}}\right] .
\end{aligned}
$$

The moment $\mathbf{N}_{d \hat{i}}$ is directed along the axis of rotation and slows rotation down; by a scalar multiplication of (9) by $\boldsymbol{\Omega}$ and, for the sake of simplicity, neglecting the very small term $\mathrm{d} \mathbf{J}_{m} / \mathrm{d} t$ we immediately get Equation (6) because

$$
\mathbf{\Omega N}_{d \|}=-\frac{2 m_{\perp}^{2} \Omega^{4}}{3 c^{3}}
$$

The torque $\mathbf{N}_{d \perp}$ is perpendicular to the angular velocity and causes the alignment of the angle $\alpha$ as

$$
\begin{aligned}
& I \frac{\mathrm{d}(\Omega \cos \alpha)}{\mathrm{d} t}=\frac{\mathbf{m} \mathbf{N}_{d}}{m}=0, \\
& I \Omega \frac{\mathrm{d} \alpha}{\mathrm{d} t}=-\mathbf{N}_{d \perp}=-\frac{2 m^{2} \Omega^{3}}{3 c^{3}}-\cos \alpha \sin \alpha, \\
& I \frac{\mathrm{d} \Omega}{\mathrm{d} t}=-\mathbf{N}_{d \|}=-\frac{2 m^{2} \Omega^{3}}{3 c^{3}} \sin ^{2} \alpha .
\end{aligned}
$$

In this case and in those described earlier the magnitude of the magnetic moment $\mathbf{m}$ is considered constant and rigidly connected to the stellar body which is rotating so that the angle $\alpha$ would decrease. The characteristic time of change of the angle $\alpha$ at $\alpha \approx 1$ is about the same as that for braking the star's rotation

$$
T_{m}=\frac{3 c^{3} I}{4 m_{\perp}^{2} \Omega_{0}^{2}}
$$

but if $\alpha \rightarrow \pi / 2$ the alignment of the moment $\mathbf{m}$ is considerably slower (at $\alpha=\pi / 2$, obviously, $\mathrm{d} \alpha / \mathrm{d} t=0)$. Articles [51, 52] contain the analysis of Equation (11). Unfor- 
tunately, the torque $\mathbf{N}$ depends both on the radiation power, which means on the plasma parameters outside the star, and on the quasistatic component of the dipole $\mathbf{m}_{\|}$. The situation is even more complicated for a non-dipole field. The same may be said if we take into account the possibility of a non-spherical shape of the star due to the presence of a solid crust or to other reasons [52a]. As a result the question of a change of the angle $\alpha$ or, what is the same, of the projection of the radiating magnetic moment $\mathbf{m}_{\perp}$ for pulsars remains open.

From observations the impression arises $[54,55]$ that for the pulsar NP 0532 in the Crab Nebula the angle $\alpha$ is close to $\pi / 2$, i.e. the magnetic dipole is almost at right angles to the rotation axis. There is apparently an analogous situation as far as ordinary magnetic stars are concerned [56]. The corresponding reasons are not yet clear but in principle, because of the plasma influence and the non strictly dipolar character of the field, the angle $\alpha$ can either not decrease or it may even increase rapidly to $\pi / 2$. So it is too early to maintain that there are some contradictions in the model of the oblique rotator.

Summarizing the above we may ascertain that the model of the oblique rotator, in that approximation, when the plasma outside the star is teneous enough and affects the radiation of the star only slightly ('vacuum approximation'), can explain a whole number of peculiarities of pulsars, namely:

the braking of the stellar rotation (increase of the period) in a time $T=10^{3}-10^{8} \mathrm{yr}$; the appearance in pulsars of a plasmatic atmosphere as a result of the presence near a rotating magnetized neutron star of an electric field inducing the outflow and acceleration of particles. The presence of a considerable extensive plasma atmosphere is supposed in the majority of models for the radiating regions of pulsars.

In the discussed 'vacuum approximation' the magnetic field at the surface of the star is $H_{0} \approx 10^{12}-10^{13} \mathrm{Oe}$, while the energy lost by the star is carried to a great extent by the outflowing plasma that also contains particles with rather high energies. The latter result is favourable, in principle, from the point of view of the possibility to explain activity in supernova remnants and some peculiarities of these remnants (specifically, we have in mind the pulsar NP 0532 in the Crab Nebula) as well as the effective acceleration of particles near pulsars (see, for example, [36, 57, 58]).

At the same time the applicability of 'vacuum approximation' remains vague, to say nothing of the fact that one has not yet solved the fundamental task of a selfconsistent determination of the parameters of the plasma and the field near a rotating neutron star - oblique rotator. That is why the evaluation $H_{0} \approx 10^{8}-10^{9}$ Oe for the field at the surface of the star does not seem improbable. The question remains unsolved of the change with time of the angle $\alpha$ between the magnetic moment $\mathbf{m}$ and the rotation axis $\Omega$. The same may be said about the estimations of the intensity of cosmic rays and nonrelativistic plasma emitted from pulsars, not to mention the intensity of their gravitational radiation. Meanwhile, in the literature devoted to pulsars it sometimes occurs that many hypothetical or only probable assumptions are considered as quite real (an example may be the assertions of a highly intense gravitational radiation of pulsars and the statement about the fundamental role of 
pulsars as sources of cosmic rays in the Galaxy; see [59]). Of course, this situation is to a considerable extent a natural reaction to such a great discovery as that of the pulsars. But irrespective of the motives we should bear in mind that the establishment of a reliable theory of a pulsar atmosphere and magnetosphere will still require hard work.

\section{Mechanisms of Pulsar Emission*}

Apart from the information on pulsars that can be obtained from the data on supernova remnants (see, for example, [60], we get all the information by analysing the radiation emitted by pulsars. Thus it is obvious that the questions concerning the mechanisms of pulsar emission and the structure of their emitting regions are of primary importance.

The first important conclusion which can easily be made on the basis of an estimation of the brightness temperature $T_{b}$ for the radio emission of pulsars is that this radio emission mechanism cannot be incoherent.

We should recall that for incoherent mechanisms of emission if there is neither absorption nor self absorption (absorption by the radiating particles themselves), the total radiative power (luminosity) $L$ of a source of radiating particles (molecules, atoms, electrons) is equal to the sum of the intensities of the separate particles. In other words, for incoherent mechanisms the power is $L=\mathscr{N} u$, but when absorption and self absorption are taken into account $L \leqslant \mathscr{N} u$, where $u$ is the power of radiation from a single particle and $\mathscr{N}$ is the total amount of radiating particles in a source. However, in a whole series of cases it is also necessary to examine the coherent mechanisms of radiation in which the intensity $L>\mathscr{N} u$ and, generally speaking, not proportional to $\mathscr{N}$. Cosmic masers on some $\mathrm{OH}$-lines and other molecules, some components of the sporadic radio emission from the Sun and the radio emission of pulsars may serve as an example.

The radiation flux emitted by a sphere of radius $r$ and observed at a distance $\mathscr{R}$ is equal to $F(v)=\left(2 \pi v^{2} / c^{2}\right) \kappa T_{b}(r / \mathscr{R})^{2}$. So the brightness temperature of the source is equal to

$$
T_{b}=\frac{c^{2} F(v)}{2 \pi k v^{2}}\left(\begin{array}{c}
\mathscr{R} \\
r
\end{array}\right)^{2}=1.04 \times 10^{13} v^{-2}\left(\frac{\mathscr{R}}{r}\right)^{2} \tilde{F}(v),
$$

where $\tilde{F}(v)$ is measured in flux units: f.u. $=10^{-26}$ watt $\mathrm{m}^{-2} \mathrm{~Hz}^{-1}$. The expression (12) may be assumed as the definition of $T_{b}$ and then it is formally applicable also outside the limits of the condition $h v \ll \kappa T_{b}$. Under this condition formula (12) holds also for the equilibrium radiation when $T_{b} \leqslant T$.

For the pulsar NP 0532 in the Crab Nebula the flux, averaged over time, is by orders of magnitude equal to

$$
\tilde{F}\left(10^{8} \mathrm{~Hz}\right) \approx 10 \text { f.u., } \tilde{F}\left(10^{15}\right) \approx 10^{-2}, \quad \tilde{F}\left(10^{18}\right) \approx 10^{-4} .
$$

\footnotetext{
* In detail see $[48,61,62]$ and the literature cited there.
} 
Hence, at $R=1500 \mathrm{pc}$ and $r=5 \times 10^{7} \mathrm{~cm}$, we get

$$
T_{b}(\text { radio }) \approx 10^{26}, \quad T_{b}(\text { optics }) \approx 10^{9}, \quad T_{b}(\text { X-rays }) \approx 10 \mathrm{~K} .
$$

The luminosity of the pulsar $L \approx 4 \pi \mathscr{R}^{2} \int F(v) \mathrm{d} v$ is of the order of $L$ (radio) $=10^{31}$, $L$ (optics) $\approx 10^{34}$ and $L$ (X-rays) $\approx 10^{36} \mathrm{erg} \mathrm{sec}^{-1}$. Even when decreasing the radius $r$ by an order of magnitude, $T_{b}$ (optics) $\approx 10^{11}$, which corresponds to a particle energy $E \approx \kappa T_{b} \approx 10^{7} \mathrm{eV}$. Hence, it is clear that the optical and X-ray emission from pulsars may be fully incoherent, for example, it can be synchrotron radiation or inverse Compton scattering. By contrast, it is evident that even at $T_{b} \approx 10^{20}$ (for NP 0532 this corresponds to a radius $r \approx 5 \times 10^{10} \mathrm{~cm}$ ), the radio emission cannot be due to incoherent mechanisms since the acceleration of a very large number of electrons up to energies $E \gtrsim 10^{16} \mathrm{eV}$ seems completely unreal (in addition to that the flux and, respectively, the value of $T_{b}$ for pulses of pulsars are considerably higher as compared with the utilized mean values). The same may be said about the $\mathrm{OH}$ sources with $T_{b} \approx 10^{12}$ and certain solar radio bursts. Thus some coherent mechanism should really be responsible for the radio emission of pulsars.

There are two essentially different types of coherent mechanisms of radio emission which may be called 'maser' and 'antenna' or 'aerial' type mechanisms.

A maser mechanism acts already in a uniform medium without previous spatial bunching of the particles. It does not also require the bunching (phasing) of particles in velocity space. Thus a maser mechanism can begin to operate in the absence of macroscopic currents varying with the radiated frequency. Maser mechanisms are analogous to self absorption. In both these cases the intensity along the path $l$ in a uniform medium varies according to the law $I=I_{0} \exp (-\mu l)$ (for self absorption, $\mu>0$ and for amplification $\mu<0$ ).

For the antenna type of radiation mechanisms the spatial nonuniformity of the source or the current distribution in the source is essential. In the simplest case we have a source, that consists of bunches of particles, one of its dimensions being $d \ll \lambda$ ( $\lambda$ is the wavelength in the medium). If all the dimensions of the bunch satify this condition, its radiation is coherent in all directions in the sense that all the particles in the bunch radiate in phase. Therefore, the total power of radiation $L_{b}=n_{b}^{2} u$, where $u$ is the radiative power of one particle and $n_{b}$ is the number of particles in the bunch. If, for example, there is an electron bunch with $d \ll \lambda$, the total power of radiation, say at its acceleration, is proportional to $\left(e n_{b}\right)^{2}$ where the radiative power of an electron is proportional to $e^{2 *}$.

* To eliminate confusion in the terminology we note the following. Radiation is called coherent, in general, when the phase of the field is fixed. Obviously, any fixed, regular (nonstatistical) current distribution radiates coherently. A particular case of such coherent radiation is the above mentioned radiation provided that the difference in phases between radiators in the bunch is small. $\mathrm{A}$ set of coherent radiators (bunches) with independent (random) phases yields, on the whole, incoherent or partially coherent radiation. This is also true for the maser radiation in cosmic conditions (and, generally, without a resonator) when the radiation from a whole source is incoherent (we mean random phases of the field in different directions and at different frequencies). That is why we carefully determine in the text the coherent radiation from coherent mechanisms of radiation defined by the condition $L>\mathscr{N} u$. However, the base of such mechanisms is some coherence, for example, within the bunch or when the waves are amplified in a given direction. 
For a source with $N$ particles and $N_{b}$ independently (incoherently) radiating bunches, it is evident that

$$
L=N_{b} n_{b}^{2} u=n_{b} N u
$$

Hence in this case the radiative power is $n_{b}$ times the value for the incoherent source with the same values of $N$ and $u$.

For the filament-shaped bunches with a diameter $d \ll \lambda$ or discs with a thickness $d \ll \lambda$, the radiation from all particles in the bunch has equal phases generally speaking, only in the direction perpendicular to the filament's axis or the disc plane. These cases are similar to thin antennae (aerials) of the proper shape. That is why we call such a coherent mechanism an antenna-type mechanism.

If the characteristic size, $d$, of the bunch increases, the intensity of radiation begins to fall rapidly as soon as $d \gtrsim \lambda$. Actually, the intensity of radiation with wave vector

$$
\mathbf{k}=\begin{gathered}
2 \pi \mathbf{k} \\
\lambda \mathbf{k}
\end{gathered}
$$

is proportional to $I \approx\left|\int j(\mathbf{r}) \exp (i \mathbf{k r}) d \mathbf{r}\right|^{2}$, where $j(\mathbf{r})$ is the current density in the source (bunch). If we restrict ourselves (for the sake of simplicity) to a one-dimensional distribution we see for the continuous current distribution of the type $j=j_{0} \pi^{-1 / 2} \times$ $\times \exp \left(-x^{2} / d^{2}\right)$ that the intensity is less than at $d \ll \lambda$ by a factor $f=\exp \left(-\pi^{2} d^{2} / \lambda^{2}\right)$. The mentioned factor $f$ is rather small already at $d=\lambda$, when $f=e^{-\pi^{2}} \approx 10^{-4}$; it is obvious that if $d=3 \lambda, f \approx 10^{-40}$ and, consequently, the antenna mechanisms are effective only at $d<\lambda$. The use of expressions like (15) is limited also by the condition of incoherence of the individual bunches. In general, it is a characteristic of the antenna mechanisms that the currents or the electromotive forces are fixed, the mutual effects of neighbouring bunches (antennas) being out of consideration.

It is extremely difficult to satisfy such requirements for meter and shorter wavelengths in cosmic conditions. First, though different mechanisms of plasma instability and some other processes induce the appearance of non-homogeneities, the letter are generally not clearly pronounced (in other words, the depth of a charge density modulation is small). Secondly, having been formed, some clearly defined bunches would, generally speaking, dissipate very quickly. The point is that in cosmic conditions it is difficult to expect the formation of monoenergetic particles and, therefore, the particles in bunches will have a marked velocity spread $\Delta v$. Thus, say, along the magnetic field, the bunch is considerably smeared at a time $\tau \approx d / \Delta v_{\|}$. Hence, for example, with $d \approx 30 \mathrm{~cm}$ and the velocity spreading along the field $\Delta v_{\|} \approx 3 \times 10^{9}$ the time $\tau \approx 10^{-8}$ sec. A bunch directed across the magnetic field (in the azimuth) is also smeared in the time

$$
\tau \lesssim \frac{2 \pi r_{H}}{\Delta v_{\perp}}=\frac{2 \pi v_{\perp}}{\Delta v_{\perp} \omega_{H}}, \quad \omega_{H}=\frac{e H}{m c} \cdot \frac{m c^{2}}{E}
$$


Even with $\Delta v_{\perp} \approx 10^{-2} v_{\perp}$, the time $\tau \lesssim 10^{3} / \omega_{H}$ and if $E / m c^{2} \approx 10^{2}, H>10^{6}$, the time $\tau<10^{-8}$ sec. We can give a lot of similar examples testifying that any pronounced antenna mechanism is unrealistic in cosmic conditions. Meanwhile, in connection with the discussion of the nature of pulsar radiation it has often been suggested in the literature to use the antenna mechanisms. However, no concrete arguments for the origin of distinct bunches and their stabilization are given. In view of this fact, the corresponding calculations appear to us completely groundless. This is true even for the radio-frequency band. As for the optical and X-ray regions it is much more difficult to speak on the occurrence of bunches or current layers with a characteristic size (diameter, thickness of the layer) $d \lesssim \lambda$. It may be, that the trend to use antenna mechanisms is connected with the fact that the radio emission from pulsars, as was said above, cannot be incoherent and at the same time the coherent maser mechanisms are not yet so well known as the classical antenna mechanisms. Nevertheless, for cosmic conditions the coherent maser mechanisms of radiation are of much greater significance than the antenna mechanisms.

It has already been mentioned above that maser coherent mechanisms of radiation act also in a uniform medium, not to mention that it is necessary to assume the medium (the radiating region) to be limited in space. True, in maser mechanisms for nontransverse (in particular, longitudinal) waves some noncompensated charges with the density $\varrho(\mathbf{r})$ appear in accordance with the equation $\operatorname{div} \mathbf{E}=4 \pi \varrho$. These charges as well as those associated with any fluctuations of concentrations are essential for the transformation of waves, for example, for the conversion of plasma (longitudinal) waves into electromagnetic (transverse) waves. However, in such cases the nonuniformities of the electron or ion concentrations have nothing in common with the discrete bunches of charges usually considered in antenna mechanisms of radiation.

Next we should like to make some remarks on maser mechanisms using the transfer equation for the intensity of electromagnetic waves $I$ :

$$
\mathrm{d} I / \mathrm{d} x=A+\left(B-\mu_{c}\right) I .
$$

If we are interested in the polarization of the radiation, analogous equations should be written also for the other Stokes parameters (see, for example, [63]). Furthermore, the refraction is neglected in (16) and below the system is assumed uniform over a path $l$ along the ray ( $x$-axis). Under such conditions the intensity of the radiation from a source in the $x$-direction will be

$$
I=\frac{A}{\mu_{c}-B}\left\{1-e^{\left(B-\mu_{c}\right) l}\right\}
$$

In (16) and (17) the coefficient $A$ corresponds to the spontaneous radiation, $B$ to the induced (maser) radiation $(B>0)$ or selfabsorption (for $B<0$ ), and $\mu_{c}$ is the absorption coefficient that is not connected with the radiating particles. In practice, for radio waves $\mu_{c}$ is the absorption coefficient due to collisions. When the magnetic field effect 
for the hydrogen plasma is neglected, we have [49]

$$
\begin{aligned}
& \mu_{c}(\omega)=\frac{1-\tilde{n}^{2}(\omega)}{c \tilde{n}(\omega)} v_{\mathrm{eff}}, \quad \tilde{n}^{2}(\omega)=1-\frac{\omega_{e}^{2}}{\omega^{2}}, \quad \omega_{e}^{2}=\frac{4 \pi e^{2} n_{e}}{m} \\
& \omega^{2} \gg v_{\mathrm{eff}}^{2}, \quad \tilde{n}^{2} \gg \frac{\omega_{e}^{2}}{\omega^{2}} \cdot \frac{v_{\mathrm{eff}}}{\omega} \\
& v_{\mathrm{eff}}=\pi \quad e^{4} \quad \sqrt{\frac{8 k T_{e}}{\pi m}} n_{e} \ln \left(0.37 \frac{k T_{e}}{e^{2} n_{e}^{1 / 3}}\right)=\frac{5.5 n_{e}}{T_{e}^{3 / 2}} \ln \left(220 \frac{T_{e}}{n_{e}^{1 / 3}}\right) .
\end{aligned}
$$

For the synchrotron radiation of electrons with an isotropic distribution in the vacuum $A=\varepsilon$ and $B=-\mu_{r}<0$. The expressions for the emissivity $\varepsilon$ and the reabsorption coefficient $\mu_{r}$ are well known [63]. Amplification (the maser effect, $B>0$ ) of synchrotron radiation may take place either if the refractive index $\tilde{n}$ in the radiating region is different from unity or in the case of an anisotropic velocity distribution of relativistic electrons. The index $\tilde{n} \neq 1$ and, specifically, $\tilde{n}(\omega)=\sqrt{1^{-\omega^{2}} / \omega^{2}} \approx 1-\left(\omega_{e}^{2} / 2 \omega^{2}\right)$ in the presence of a 'cold' plasma with a number density $n_{\boldsymbol{\epsilon}}$ in the source (we assume that

$$
\left.\omega_{e}^{2}=\frac{4 \pi e^{2} n_{e}}{m} \ll \omega^{2}\right) .
$$

The anisotropy of the electron velocity distribution leads to amplification if this anisotropy is significant already in the interval of angles

$$
\eta=\sqrt{\left(\frac{m c^{2}}{E}\right)^{2}+\omega_{e}^{2}} \approx \sqrt{1-\tilde{n}^{2} \beta^{2}}, \quad \beta=\frac{v}{c}
$$

(see $[62,63]$ and the literature cited there).

If a longitudinal (plasma) wave with frequency $\omega_{l} \approx \omega_{e}$ and intensity $I_{l}$ is propagating in the plasma, transverse (radio) waves with the frequencies $\omega \approx \omega_{l}$ are produced due to the processes of spontaneous and induced scattering. In (16) in this case $\mathscr{A}=\alpha I_{l}$ and $B=\beta I_{l}$ (the expressions for $\alpha$ and $\beta$ see in [48]). In this case the amplification of radio emission is high if $\left(\beta I_{l}-\mu_{c}\right) l \gg 1$. For such mechanisms it is easy to obtain the necessary radio luminosity and the brightness temperature $T_{b}$ for pulsars.

The conversion of a plasma wave into radio emission is a particular case of the processes of transformation (due to scattering and, generally, because of the nonlinearity of the plasma) of one type of normal waves into another which can propagate in this plasma. When the magnetic field is present these waves are, in general, neither transverse nor longitudinal [49, 64]. In addition to spontaneous and induced transformation of different waves, these waves are generated, amplified and absorbed in the plasma as a result of a whole series of processes (streams of particles, shock waves). Due to this, the plasma turbulence, represented in some approximation by a set of different normal waves, produces electromagnetic radiation exciting from the plasma $[49,64,65]$; when $\left(\beta I_{l}-\mu_{c}\right) l \gg 1$ this radiation is just the maser radiation. 
The third important class of maser coherent mechanisms acts in the simultaneous presence of plasma turbulence and relativistic particles [65]. In principle, this mechanism is especially similar to the inverse Compton scattering of electromagnetic waves in vacuum on relativistic electrons (more exactly, the maser effect is associated only with induced scattering; the spontaneous scattering of the plasma turbulence on relativistic particles is also of interest).

Let a wave (frequency $\omega_{1}$, wave vector $\mathbf{k}_{1} ; k_{1}=\left(\omega_{1} / c\right) \tilde{n}\left(\omega_{1}\right)$ scatter on a relativistic electron with velocity $\mathbf{v}$, and transform into a wave with frequency $\omega_{2} \equiv \omega$ and wave vector $\mathbf{k}_{2} \equiv \mathbf{k}$. The wave types 1 and 2 can be different but always

$$
\omega_{1}-\mathbf{k}_{1} \mathbf{v}=\omega-\mathbf{k v},
$$

where the energy loss of the electron due to the scattering is assumed small*. The condition (19) can be rewritten as

$$
\omega\left[1-\frac{v}{c} \tilde{n}(\omega) \cos (\mathbf{k v})\right]=\omega_{1}\left[1-\frac{v}{c} \tilde{n}\left(\omega_{1}\right) \cos \left(\mathbf{k}_{1} \mathbf{v}\right)\right]
$$

and the frequency $\omega$ is maximum at $\cos \left(k_{1} \mathbf{v}\right)=-1, \cos (\mathbf{k v})=1$ (frontal collision), that is

$$
\omega \leqslant \omega_{\max }=\frac{\omega_{1}\left(1+(v / c) \tilde{n}\left(\omega_{1}\right)\right)}{1-(v / c) \tilde{n}(\omega)} \leqslant \frac{2 \omega_{1}}{1-(v / c) \tilde{n}(\omega)} .
$$

If the frequency is so high that one may put $\tilde{n}(\omega)=1$,

$$
\omega_{\max } \leqslant 4 \omega_{1}\left(\frac{E}{m c^{2}}\right)^{2}
$$

since for relativistic particles

$$
1-\frac{v}{c} \approx \frac{1-v^{2} / c^{2}}{2}=\frac{1}{2}\left(\frac{m c^{2}}{E}\right)^{2} .
$$

We should like to note that it is also possible to treat synchrotron radiation as the frequency conversion - in this case in (20) we should put $\omega_{1} \approx e H / m c$ (for details see $[62,67])$. For inverse Compton scattering in the vacuum, the role of $\omega_{1}$ is played by the frequency of a scattered soft photon. In the nonrelativistic plasma, the frequency of normal waves $\omega_{1}$ is defined by the characteristic frequencies

$$
\omega_{e}=5.64 \times 10^{4} \sqrt{n_{e}}, \quad \omega_{i}=\sqrt{\frac{4 \pi e^{2} n_{e}}{m_{i}}}=\frac{\omega_{e}}{43}
$$

* Condition (19) is most easily obtained on the basis of the quantum representation in view of which a photon in the medium (plasmon, etc.) has the energy $h \omega$ and the momentum $\hbar \mathbf{k}$ (see [66]). The conservation laws for energy and momentum in the scattering process take the form: $E+\hbar \omega_{1}=E_{2}+\hbar \omega_{2}, \mathbf{p}_{1}+\hbar \mathbf{k}_{1}=\mathbf{p}_{2}+\hbar \mathbf{k}_{2}$, where $E=\sqrt{ }\left(m^{2} c^{4}+c^{2} p^{2}\right)$ is the energy of a particle. Hence, for small changes of the energy $E_{2}-E_{1}=h\left(\omega_{2}-\omega_{1}\right)=(\partial E / \partial \mathbf{p}) \Delta \mathbf{p}=\mathbf{v} \Delta \mathbf{p}=h \mathbf{v}\left(\mathbf{k}_{2}-\mathbf{k}_{1}\right)$ which leads to (19). 
(for the hydrogen plasma considered) and $\omega_{H}=e H / m c=1,76 \times 10^{7} H$. We should have in mind also the possibility of different mechanisms to operate simultaneously when $A$ and $B$ in (16) represent the sum of emissivity and the sum (due to all mechanisms) of amplification or selfabsorption. In particular, the synchrotron radiation can make the main contribution to $A$ but the amplification can be determined by plasma turbulence.

Let us also refer to the possibility of obtaining the maser mechanism in a dense plasma placed in a strong magnetic field $\left(H \gtrsim 10^{8} \mathrm{Oe}\right)$ and spontaneously radiating due to collisions ('one-dimensional' bremsstrahlung radiation) and transitions between lower magnetic levels (cyclotron radiation) [68]. However, it is not clear yet whether the radiation in this case may escape from the source. The possibility of obtaining inverse level population when the necessary account taken of electron collisions also seems unreal.

The possibility of a sharply directional and polarized radiation occurring is characteristic for maser mechanisms. The point is simply that the amplification coefficient $e^{B l}$ at $B l \gg 1$ is very sensitive to the value $B l$ which in turn depends on the path length, wave polarization and other parameters. Therefore, the values of $B l$ can easily be distinguished from each other in different directions and for different polarizations. Obviously, this particular feature of the maser mechanisms is highly favourable from the point of view of interpreting the radio emission from pulsars. The main thing, however, is that the maser mechanisms, in the sense of their effectiveness, can produce radiation with as high brightness temperature as needed. Finally, since the applicability of the antenna mechanisms in cosmic conditions seems unrealistic, the use of one or the other maser mechanism for interpreting the pulsar radio emission seems inevitable.

\section{Some Models of Radiating Regions of Pulsars}

The centre of gravity of the question of pulsar radiation lies in the choice of models for radiating regions because there is no difficulty either so far as potentialities of different mechanisms of radiation are concerned or from the energetic point of view. On the contrary, even such fundamental questions as the character of polar diagrams of radiation remain obscure (we mean the choice between a 'pencil-beam' and a 'knife-like' or 'fan-beam' diagram; see below), as well as the characteristic length $l$ and the distance $r$ of the radiating regions from the surface of the star. We are also ignorant of the distribution function of plasma particles in radiating regions, and its determination from the data on the radiation itself is of considerable obscurity (to say nothing of the momentum distribution function of particles even the determination of such integral parameters of the plasma like the number densities of the radiating ultrarelativistic particles $n_{r}$, their mean energy $E$, the density of 'cold' plasma $n_{e}$, its temperature $T_{e}$ and others are not unique.)

In order that the radiation of a rotating star can be observed as comparatively short pulses (the duration of a pulse $\delta P \ll P$ where $P$ is the pulsar period), the polar diagram of the radiation with a characteristic angle $\Delta \varphi$ must b sufficiently narrow 
(models of rotating sources with such a diagram are often called the 'lighthouse' models).

Obviously,

$$
\Delta \varphi \approx \frac{2 \pi}{P} \delta P=\Omega \delta P .
$$

For NP 0532 the angle $\Delta \varphi \approx 20-30^{\circ}$ while for the majority of other pulsars the angle $\Delta \varphi$ is less than that.

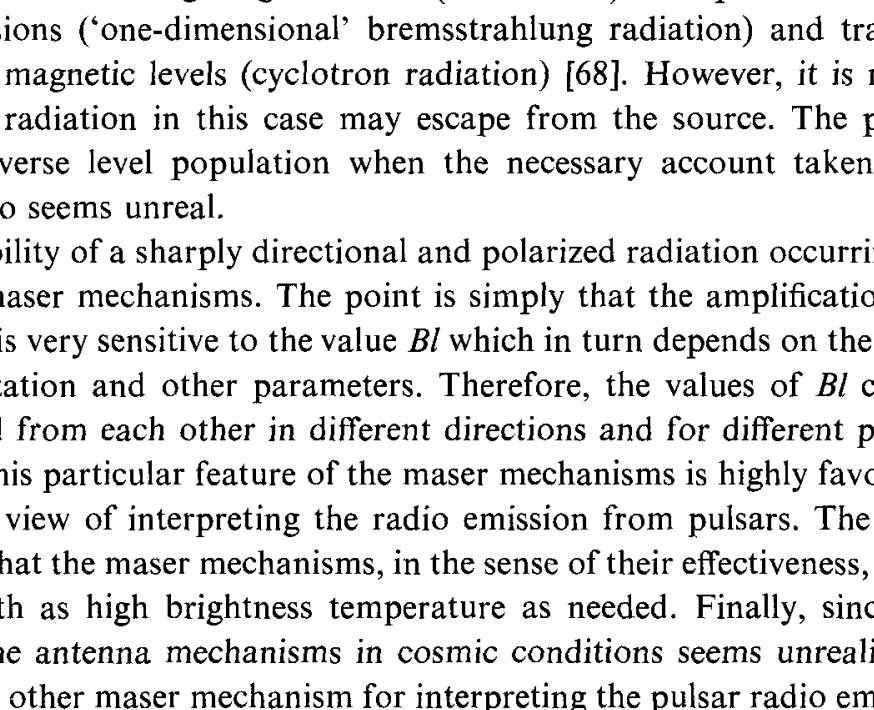

Fig. 3. A 'pencil-beam' polar diagram (a cross-section). The diagram has axial symmetry around the magnetic axis.

If the angle $\Delta \varphi$ defines the aperture of the diagram in all directions we deal with a 'pencil-beam' polar diagram; its axis can, for example, coincide with that of magnetic symmetry (the direction of the dipole $\mathbf{m}$; see Figure 3). The other typical possibility is a 'knife-like' polar diagram when the angle $\Delta \varphi$ defines only the least possible aperture of the diagram while in the perpendicular direction the aperture is defined by the angle $\varphi_{\perp} \approx 1$ or even $\varphi_{\perp}=2 \pi$. Such a diagram corresponds to the case, for example, when the radiation is concentrated in the angle $\Delta \varphi$ near the equatorial plane of the magnetic star (Figure 4).

The solid angles held by pencil-beam (pb) and a knife-like $(K b)$ diagrams have the order of magnitude $\Delta \Sigma_{p b} \approx(\Delta \varphi)^{2}$ and $\Delta \Sigma_{k b} \approx 2 \pi \Delta \varphi$ (at $\varphi_{\perp} \approx 2 \pi$ ). Further at every revolution of a 'lighthouse' (star) the diagrams draw on the celestial sphere solid angles

$$
\Delta \Sigma_{p b}^{(s)} \approx 2 \pi \sin \alpha \cdot \Delta \varphi, \quad \Delta \Sigma_{k b}^{(s)} \approx 4 \pi \sin \alpha .
$$

For an isotropic source and by order of magnitude also for a dipole source $\Delta \Sigma_{0}^{(s)} \approx 4 \pi$. So if $\sin \alpha \approx 1$ in the case of a knife-like diagram the pulsar is 'seen' from almost any 


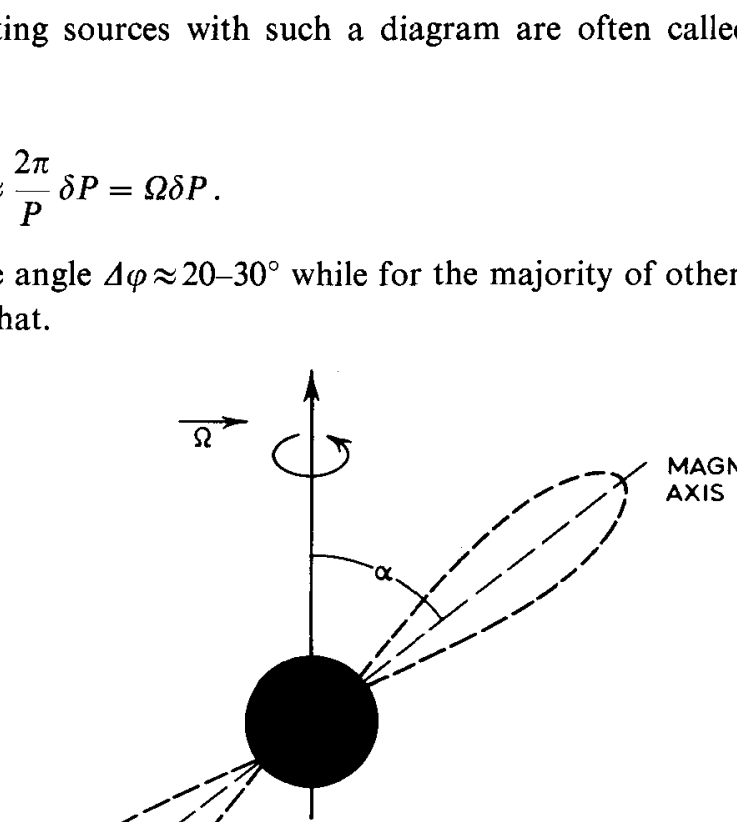

Fig. 4. A 'knife-like' polar diagram (cross-section). The diagram has axial symmetry around the magnetic axis.

direction and the estimated pulsar's concentration is the same as for an isotropic source (all stars except pulsars are practically isotropic sources). In the case of a 'pencil-beam' diagram the ratio

$$
\frac{\Delta \Sigma_{p b}}{\Delta \Sigma_{0}^{(s)}} \approx \sin \alpha \cdot \Delta \varphi
$$

and the concentration of pulsars is $1 /(\sin \alpha \cdot \Delta \varphi)$ times the estimation for isotropic sources. If the pulsar NP 0532 in the Crab Nebula has a pencil-beam diagram we can observe it only due to the lucky circumstance that the diagram axis appears to be near the pulsar-Sun line.

For a knife-like diagram with the angle $\varphi_{\perp}=2 \pi$, generally speaking, two pulses should be observed during a pulsar period [61, 69]*. This takes place for NP 0532 and, therefore, the assumption of a knife-like character of the diagram meets no objection. But the same picture can be observed also for pencil-like diagrams. Besides different non-symmetric diagrams can exist, for example, a knife-like one with $\varphi_{\perp}<2 \pi$.

* Let the direction of observation make the angle $\Psi$ with the rotation axis (Figure 5). Then if $\alpha<\pi / 2$ and $\pi / 2-\alpha<\Psi<\pi / 2$ two non-equidistant pulses would be observed in a sidereal revolution (at $\Psi<\pi / 2-\alpha$ there is no radiation). If $\alpha=\pi / 2$ these pulses are equidistant and the pulsar period $P=\pi / \Omega=P_{s t} / 2$, where $P_{s t}=2 \pi / \Omega$ is the star's rotation period. If $\alpha \neq \pi / 2$ one pulse for a pulsar period would be observed at $\Psi=\pi / 2$ (two pulses in a sidereal revolution, i.e. $P=P_{s t} / 2$ ) and at $\Psi=\pi / 2-\alpha$ (in this case $\left.P=P_{s t}\right)$. 


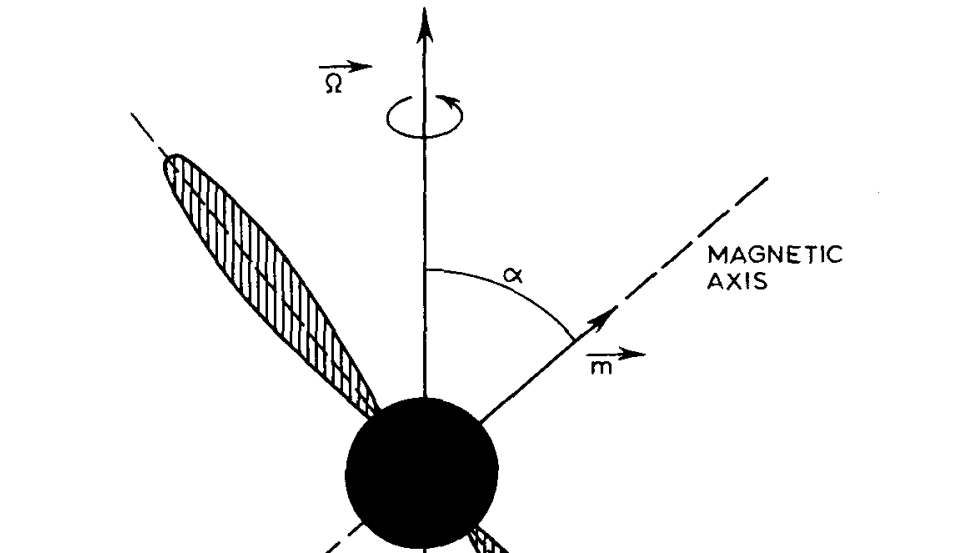

Fig. 5. A 'knife-like' polar diagram (explanation).

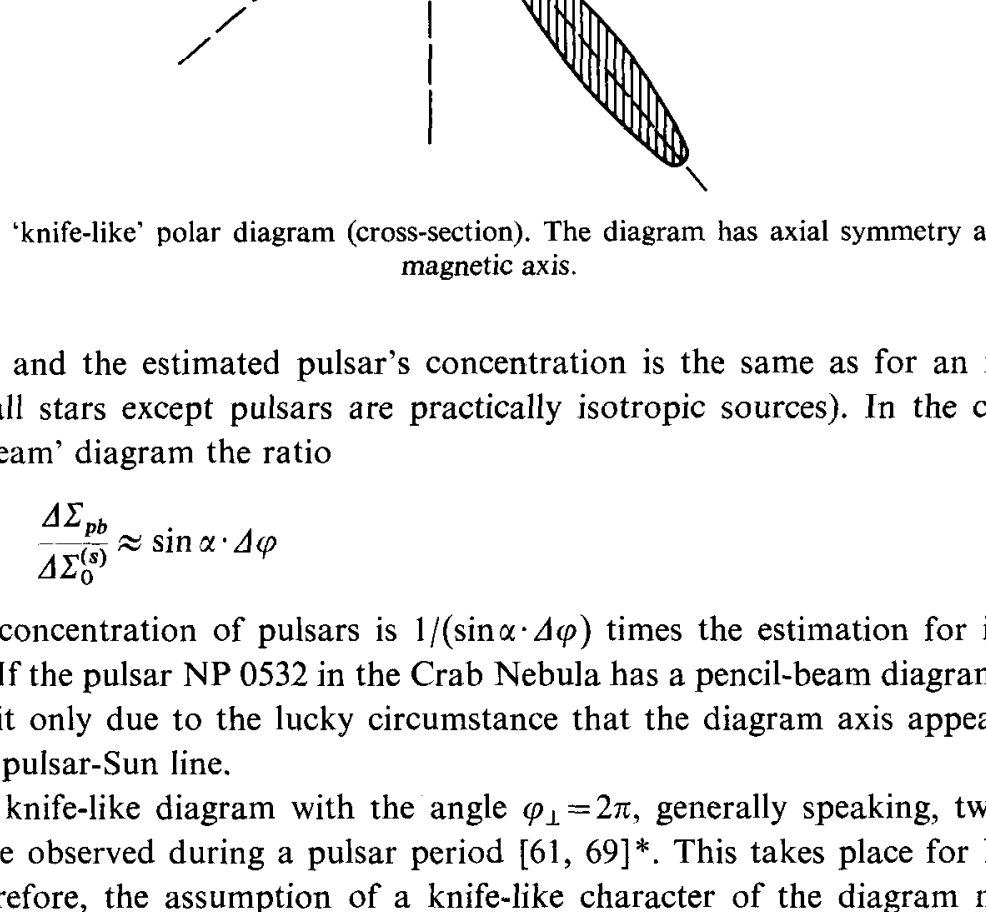

Fig. 6. A model of the radiating regions of pulsars located in polar regions (a 'pencil-beam' diagram).

For the pulsar in the Crab Nebula, apparently, $\alpha \approx \pi / 2$ (see $[54,55]$ ). In the following estimates of the characteristic size of the radiating regions $l$ for a knife-like diagram, we deal with the thickness of the ringlike radiation belt in the plane of the magnetic equator, while for a pencil-beam diagram we speak of the diameter of the radiation 'cap' over the magnetic poles (Figure 6). We will consider the optical and $\mathrm{X}$-ray radiation of this pulsar as incoherent synchrotron radiation. Such a hypothesis 
seems most probable $[48,58,61,52]$ because the high effectiveness of the synchrotron mechanism is known from a number of examples. Besides, alternative possibilities (radiation from a dense plasma [68], inverse Compton scattering of radio photons on relativistic electrons $[71,72]$ and the scattering of plasma waves on relativistic electrons with their transformation into radio waves [73]) meat some objections.*

It is not at all difficult to make synchrotron models of the infrared, visible (optical) and X-ray emission of the pulsar NP 0532 that describe in detail the form of a given spectrum. Such models, however, are not unique since the question of the parameters of the radiating regions (their form, configuration of the field and so on) is open. Besides, we should consider a self-consistent model, that is to take into account not only the radiation but also the particle acceleration, their trapping in radiation zones and so on.

In such a situation we will limit ourselves to a rough approximation, that is we will consider the electron spectrum to be quasimonoenergetic (mean energy $E$; the energy spread $\Delta E \ll E$ ). The radiation spectrum of such electrons is well known (see, for example, [67]). Taking the radiation flux $F(v)$ for the infrared, optical and X-ray frequencies $v_{i}, v_{0}$ and $v_{x}$ one can determine the frequency $v_{m}$ and the power $P\left(v_{m}\right) \equiv$ $\equiv L\left(v_{m}\right)$ in the maximum of the radiation spectrum as well as the optical depth $\tau\left(v_{i}\right)$ for an infrared frequency (the selfabsorption for higher frequencies is too small). Then the frequency

$$
v_{m}=0.07 \frac{e H_{\perp}}{m c}\left(\frac{E}{m c^{2}}\right)^{2}=1.2 \times 10^{6} H_{\perp}\left(\frac{E}{m c^{2}}\right)^{2} \mathrm{~Hz} .
$$

The radiative power at the maximum is

$$
P\left(v_{m}\right)=1.6 \frac{e^{3} H_{\perp}}{m c^{2}} n_{r} V \sim 10^{-22} H_{\perp} n_{r} l^{3} \mathrm{erg} \mathrm{sec}^{-1} \mathrm{~Hz}^{-1}
$$

The optical depth for selfabsorption [63]

$$
\begin{aligned}
& \tau(v)=\mu_{r} l=\frac{4 \pi}{3 \sqrt{3} H_{\perp}}\left(\frac{m c^{2}}{E}\right)^{5} n_{r} l K_{5 / 3}(z), \\
& z=\frac{4 \pi m c}{3 e H_{\perp}}\left(\frac{m c^{2}}{E}\right)^{2} v=0.29 \frac{v}{v_{m}} ; \quad z \ll 1, \quad K_{5 / 3}(z)=\frac{2^{5 / 3} \Gamma\left(\frac{2}{3}\right)}{3} z^{-5 / 3} .
\end{aligned}
$$

Hence, obviously, one may express $E / m c^{2}, H_{\perp}, n_{r}$ and $l \sim V^{1 / 3}$ with the help of one and the same arbitrary parameter for which we choose the ratio of the magnetic energy

\footnotetext{
* In a dense plasma it is difficult to obtain an inverse level population (it is destroyed by collisions). Inverse Compton scattering is of great interest from the point of view of the pulsar $\gamma$-radiation appearance but in all probability it is impossible to associate optical and $\mathrm{X}$-ray radiation with Compton scattering (as is clear from calculations [72]; the other author of the 'Compton model' [71] also passed on to various synchrotron models [74]). For the transformation of plasma waves into X-ray radiation by to scattering of relativistic particles the plasma wave frequencies should be improbably high (at moderate values of the ratio $E / m c^{2}$ ).
} 
density to the energy density of relativistic electrons $\delta=\left(H^{2} / 8 \pi E n_{r}\right)$ (more exactly we deal with the projected component of the field, $H_{\perp}$, perpendicular to the line of sight but below we put $H \approx H_{\perp}$ which is, of course, not obligatory). As a result we have

$$
\begin{aligned}
& H \approx H_{\perp} \approx 10^{6} \delta^{4 / 17} \mathrm{Oe}, \quad l \approx 5 \times 10^{6} \delta^{1 / 17} \mathrm{~cm}, \\
& E / m c^{2} \approx 10^{2} \delta^{-2 / 17}, \quad n_{r} \approx 5 \times 10^{14} \delta^{-7 / 17} \mathrm{~cm}^{-3}, \\
& \tau\left(y=1.36 \times 10^{14}\right)=\mu_{r} l=1.75 .
\end{aligned}
$$

Probably, for the pulsar in the Crab Nebula $\delta \gg 1$ and, in any case, $\delta \gtrsim 1$ in order that the cloud of relativistic particles should be kept near the star. It is only at $\delta \gg 1$ that the synchrotron losses substitute the Compton losses. Thus already with $\delta \approx 1 \mathrm{Comp}$ ton losses (life-time $t_{c}=10^{-7} \mathrm{sec}$ ) are nearly 10-100 times then synchrotron losses (the lifetime $t_{m}=5 \times 10^{-6} \mathrm{sec}$ )* while the intensity of Compton $\gamma$-rays $\left(\tilde{E}_{\gamma}=2 \times 10^{6} \mathrm{ev}\right.$ ) would reach $10^{37} \mathrm{erg} \mathrm{sec}^{-1}$.

In the discussed model the radiation region for light and X-rays is $l \approx 5 \times 10^{6} \mathrm{~cm}$, so it is most probable that the distance to the surface of the star will also be $r \approx 5 \times 10^{6}$ $\mathrm{cm}$. But it means that at the surface of the star $r_{0} \approx 10^{6} \mathrm{~cm}$, the magnetic field $H_{0} \approx$ $\approx\left(r / r_{0}\right)^{3} H \approx 3 \times 10^{8}$ Oe. If the field $H_{0} \approx 10^{12}$ and $r \approx 10 r_{0} \approx 10^{7} \mathrm{~cm}$ the lifetime (27) of the electrons moving at a large angle to the field (at $H_{\perp} \approx H \approx H_{0}\left(r_{0} / r\right)^{3} \approx 10^{9}$ ) will reach $t_{m} \approx 10^{-11} \mathrm{sec}$ (at $E / m c^{2} \approx 10^{2}$ ). Under such conditions, in all probability, relativistic electrons can 'survive' only if they are moving at a very small angle to the field. The character of the magnetobremsstrahlung radiation under such conditions differs considerably from ordinary synchrotronic radiation $[63,75]$ (hereby we should possibly consider also the curvature of the magnetic field lines [76]). If $H_{0} \approx 10^{12}$ for keeping the above discussed model one may take $l \ll r \approx 10^{8} \mathrm{~cm}$, i.e. we should move the radiating region to the domain of a 'light cylinder' (7).

The character and mechanism of the pulsar radio emission is not yet clear. Let us assume, for example, the radio-emission of NP 0532 to be coherent synchrotron radiation with amplification due to the presence of a 'cold' plasma. In this case for quasimonoenergetic electrons the maximum value of the amplification factor $|\mu|$ is determined by the formula (see [63])

$$
\mu=-1.6 \times 10^{-2} \frac{n_{r} H_{\perp}^{2}}{\left(E / m c^{2}\right)^{2} n_{e}^{3 / 2}} \mathrm{~cm}^{-1},
$$

where $n_{e}$ is the electron density in the 'cold' plasma (in (28) this plasma is considered non-relativistic, but generally it may be regarded also as a relativistic plasma with temperature $T_{e}$, satisfying the condition $k T_{e} \ll E$, where $E$ is the energy of the radiating ultra-relativistic electrons).

* As is well known the energy of relativistic electrons in the magnetic field decreases to one half in a time

$$
t_{m}=\frac{2 m^{3} c^{5}}{3 e^{4} H_{\perp}^{2}}\left(\begin{array}{c}
m c^{2} \\
E
\end{array}\right)=\frac{5 \times 10^{8}}{H_{\perp}^{2}} \frac{m c^{2}}{E} \sec .
$$

If $H_{\perp}=10^{6}$ and $E / m c^{2}=10^{2}$, the time $t_{m}=5 \times 10^{-6} \mathrm{sec}$. 
The flux $F(v)$ observed for NP 0532 at the frequency $v \approx 3 \times 10^{7} \mathrm{~Hz}$ can be obtained with the choice of parameters (for $\delta=H^{2} / 8 \pi E n_{r} \approx 1$ )

$$
\begin{aligned}
& H \approx H_{\perp} \approx 30 \mathrm{Oe}, \quad l \approx 10^{8} \mathrm{~cm}, \\
& E / m c^{2} \approx 8, \quad n_{r} \approx 10^{7}, \quad n_{e} \approx 3 \times 10^{8}, \quad T_{e}>10^{4} \mathrm{~K} .
\end{aligned}
$$

With this

$$
|\mu| \approx 5 \times 10^{-7} \text { and } \exp \{|\mu| l\} \approx 10^{20}
$$

If the magnetic field decreases according to the law $H \approx H_{0}\left(r_{0} / r\right)^{3}$, then for $H_{0} \approx 10^{8}$ the radioemitting region is situated at $r \approx 1-2 \times 10^{8} \mathrm{~cm}$ that is near the 'light cylinder' $r_{c}=c / \Omega \approx 1.5 \times 10^{8} \mathrm{~cm}$ (the angular velocity $\Omega=200$ ). Assuming it to have a knifelike diagram this model is schematically presented in Figure 7 . If the field $H_{0} \approx 10^{12}$ we should push the radio emitting region back to the distance $r \approx 10^{10}$, the field decreasing according to the law $\left(r_{0} / r\right)^{3}$. But this does not seem probable and at $H_{0} \approx 10^{12}$ the amplification of the radioemission is rather not due to the 'cold' plasma effect. However, also with $H_{0} \approx 10^{8}$ we have no particular reasons for considering wave amplification to be associated with the 'cold' plasma. It is by no means less probable, for example, that the amplification takes place due to an anisotropic velocity distribution of relativistic electrons (in this case the presence of a 'cold' plasma

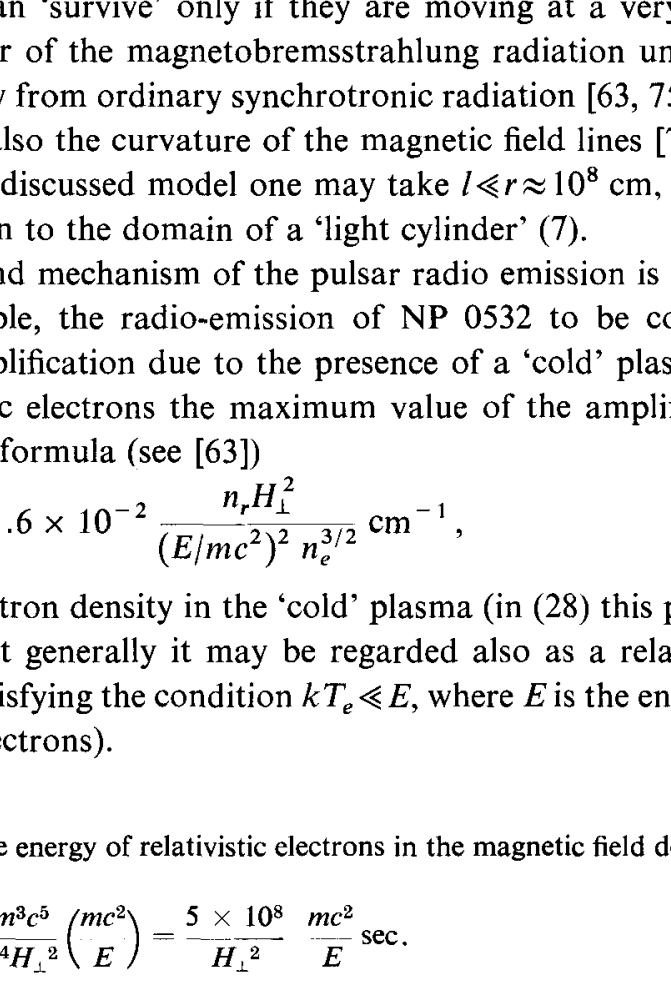

Fig. 7. A model of the radiating regions of the pulsar NP 0532 (a 'knife-like' diagram). 
is not obligatory)*. Different mechanisms for the transformation of plasma waves into radio waves can fully prove to be effective as well (see the previous chapter and [73a]). Hence we should express a great uncertainty with regard to concrete models of the pulsar radioemission. Let us mention also the delicacy of the question of the polarization of the radioemission, $[28,77]$ the analysis of which can at the same time make things clear in many respects**. We should like to emphasize also the presence of the second (short) period in pulsars which has been discovered for a number of pulsars [77a]. Apparently, in this case we deal with some oscillations or differential rotation in the radioemitting regions of pulsars but the exact nature and the character of corresponding processes are not at all clear yet.

Coherent mechanisms of radiation are obviously rather sensitive to different parameters because the amplification is determined by factors of the exponential type (the factor $\left.e^{|\mu| l}\right)$. It is quite possible also that the pulsar NP 0532 is not a typical representative even of young pulsars. In view of this it is not excluded that the ratio of fluxes in radio and optical or X-ray bands can vary within rather wide limits. Specifically, there arises the question of the possibility to record optical or X-ray pulsars not only among the observed radiopulsars $[61,78]$. Meanwhile, as far as we know there have been attempts up to now to discover pulsating optical and X-ray radiation only for radiopulsars (for this case there is an advantage, of course, of knowing the period) and for some X-ray 'stars'.

There are no lines in the spectrum of the only known optical pulsar - the one in the Crab Nebula $[79,80]$. This is to be expected for a neutron star. So it is natural to search for optical pulsars (which are not at the same time intensive enough to be observed in the radioregion) among the stars whose spectra have no lines (there are many such stars in the sky [80]).

\section{The Utilization of Pulsars in Astronomy and Physics}

The most important point in the discovery of pulsars is their probable identification with neutron stars (the discussion of the possible existence of neutron stars began already as far back as in 1934; see [81]). Apart from the study of pulsars (neutron stars) themselves, their role in supernova remnants [36, 59, 83-85] attracts attention.

Finally, the fact that sharp and strictly periodic signals escape from pulsars (the

* The latter circumstance is rather important for it may well be that due to the rapid acceleration (heating) of the particles all the plasma near a pulsar and even up to the distance $r_{c}=c / \Omega$ is relativistic or ultrarelativistic.

** The analysis of the processes of propagation and escape of the radio emission from the pulsar's magnetosphere shows that a strong linear polarization of the pulsar's radio emission can be explained only when the escape into the interstellar medium takes place in the region of quasitransversal magnetic fields. The detailed character of the polarization and its change during the pulse can be connected with the different relative position of the layer where the transformation of a quasitransversal propagation to a quasilongitudinal one takes place and the region of so-called limiting polarization (above it was assumed that the propagation is governed by the nonrelativistic plasma; if all the plasma in the magnetosphere is ultrarelativistic the situation can be different). 
secular increase of the period may be taken into account) makes them interesting for astronomy and physics. Some astronomical applications of findings related to pulsars are connected not with the periodicity of radiation but with its polarization and the point-like character or favourable location of the sources on the celestial sphere.

The propagation of radio waves in the interstellar plasma may be considered in very good approximation as 'quasilongitudinal'* and, besides, the index of refraction $\tilde{n}$ is quite close to 1 . That is why we may take that

$$
\begin{aligned}
& \tilde{n}=1-\frac{\omega_{e}^{2}}{2 \omega^{2}}=1-4.03 \times 10^{7} \frac{n_{e}}{v^{2}}, \tilde{n}_{ \pm}=1-\frac{\omega_{e}^{2}}{2 \omega\left(\omega \mp \omega_{H} \cos \theta\right)} \\
& \tilde{n}_{-}-\tilde{n}_{+}=\frac{\omega_{e}^{2} \omega_{H} \cos \theta}{\omega^{3}}=2.3 \times 10^{14} \frac{n_{e} H \cos \theta}{v^{3}} \\
& |\tilde{n}-1| \ll 1,\left|\tilde{n}_{-}-\tilde{n}_{+}\right| \ll|\tilde{n}-1|, \tilde{n}=\frac{\tilde{n}_{+}+\tilde{n}_{-}}{2},
\end{aligned}
$$

where $\tilde{n}_{-}-\tilde{n}_{+}$is the difference between the refraction indices for circularly polarized waves with different directions of the field vector rotation.

In the interstellar medium the difference $\left|\tilde{n}_{-}-\tilde{n}_{+}\right| \leqslant \omega^{2} \omega_{H} / \omega^{3}$ is very small **, therefore it should be regarded only while considering its integral effect as Faraday rotation of the polarization plane (see below), which disappears at $H=0$. With the calculating of the phase and group delay the interstellar plasma may be considered isotropic with the index $\tilde{n}=\left(\tilde{n}_{+}+\tilde{n}_{-}\right) / 2$ given in (30); with this $|\tilde{n}-1|=4.03 \times 10^{7} \times$ $\times n_{e} / v^{2} \lesssim 10^{4}$ at $n_{e} \lesssim 10^{2}$ and $v \gtrsim 10^{7} \mathrm{~Hz}$.

Inhomogeneities in the interstellar medium always satisfy the condition

$$
\frac{\lambda_{0} \mid \frac{\mathrm{d} \tilde{n} / \mathrm{d} z \mid}{2 \pi} \tilde{n}^{2}}{2 \pi} \sum_{2 \pi}^{\lambda_{0}}\left|\frac{\mathrm{d} \tilde{n}}{\mathrm{~d} z}\right| \ll 1
$$

i.e. the change of the index $\tilde{n}$ along a wavelength $\lambda_{0}=c / v$ is negligibly small. Under such conditions the phase delay for the distance $\mathscr{R}$ is with a very high degree of accuracy (see [85a]) equal to

$$
\varphi=\frac{\omega}{c} \int_{0}^{\mathscr{R}} \tilde{n}(\omega, s) \mathrm{d} s=(\omega / c) \mathscr{R}-8.5 \times 10^{-3} \frac{\int_{0}^{\mathscr{R}} n_{e} \mathrm{~d} s}{v} .
$$

* In the interstellar medium the electron density $n_{e} \approx 10^{-2}-10^{2} \mathrm{~cm}^{-3}$ and the intensity of the magnetic field $H \approx 10^{-6}-10^{-5}$. That is why $\omega_{e}=\left(4 \pi e^{2} n_{e} / m\right)^{1 / 2}=5.64 \times 10^{4} \times \sqrt{n_{e}} \approx 5 \times 10^{3}-5 \times 10^{5}$ and $\omega_{H}=e H / m c=1.76 \times 10^{7} H \approx 10-100$, i.e. $\omega_{e} \gg \omega_{H}$. Under these conditions the propagation of waves may be considered quasi-longitudinal if $\left(\omega_{H}^{2} \sin ^{4} \theta\right) /\left(4 \omega^{2} \cos ^{2} \theta\right) \ll 1$ and $\left(\omega_{H}^{2} \sin ^{2} \theta / 2 \omega^{2}\right) \ll 1$, where $\theta$ is the angle between the line of sight (the wave vector) and the field $\mathbf{H}$ (see [49], $\S 11$ ). Thus it is clear that the quasilongitudinal approximation is true even at $\omega=2 \pi \nu \sim 6 \times 10^{7}(\lambda \sim 30 \mathrm{~m})$ while the angle $\pi / 2-\theta \gg 10^{-6} \approx 0^{\prime \prime} .2$ i.c. practically always. In formula (30) in the interstellar medium the ratio $\left(\omega_{e}{ }^{2} \omega_{H} / \omega^{3}\right) \lesssim\left(3 \times 10^{13} / \omega^{3}\right) \lesssim 10^{-10}$ at $\omega \gtrsim 6 \times 10^{7}$. The absorption in (30) is not considered since for frequencies $v \gtrsim 10^{7}$ it is usually small enough.

** We refer again to the previous footnote. 
The time of the group delay of the signal is

$$
\Delta t_{g r}=\int_{0}^{\mathscr{R}} \frac{\mathrm{d} s}{v_{g r}}=\frac{\mathrm{d} \varphi}{\mathrm{d} \omega}=\frac{\mathscr{R}}{c}+\frac{1.35 \times 10^{-3} \int_{0}^{\mathscr{R}} n_{e} \mathrm{~d} s}{v^{2}} .
$$

From measurements at different frequencies one can find the value $\Delta t_{g r}(v)-\mathscr{R} / c$; so for pulsars we can immediately determine the integral quantity of electrons along the line of sight $\mathscr{N}_{e}=\int_{0}^{\mathscr{R}} n_{e} \mathrm{~d} s$ or $\mathscr{N}_{e}=\bar{n}_{e} \mathscr{R}$, since the refraction is small and the trajectory of the ray may be considered a straight line. The coefficient

$$
D M=\frac{e^{2}}{2 \pi m c^{3}} \mathscr{N}_{e}=1.5 \times 10^{-24} \mathscr{N}_{e}
$$

in the expression $\Delta t_{g r}-\mathscr{R} / c=D M \lambda^{2}$ is sometimes called the dispersion measure (if $\mathscr{N}_{e}$ is measured in particles per pc $\cdot \mathrm{cm}^{-3}$ and $\lambda$ in meters, $D M=5.8 \times 10^{-2} \mathscr{N}_{e} \mathrm{sec}$ $\mathrm{m}^{-2}$; some authors call the value of $\mathscr{N}_{e}$ itself the dispersion measure.) If we consider the change of the particle density distribution with time the determined value $\mathscr{N}_{e}=$ $=\int n_{e} \mathrm{~d} s=\int n_{e}\left(s, t_{g r}\right) \mathrm{d} s$, where $n_{e}\left(s, t_{g r}\right)$ is the density at the point $s$ and the moment $t_{g r}$ when the pulse considered passes this point.

The use of the value $\mathscr{N}_{e}$ obtained from the data on the delay time for pulsars with the addition of other information gives us the possibility of getting valuable knowledge of the interstellar medium. Thus, in the galactic plane according to [86] $\bar{n}_{e}=$ $=0.05 \mathrm{~cm}^{-3}$ in contrast to the value $\bar{n}_{e}=0.1 \mathrm{~cm}^{-3}$ accepted previously. We should note that the values of $\mathscr{N}_{e}$ for known pulsars are approximately $\gtrsim 3 \mathrm{pc} \cdot \mathrm{cm}^{-3} \approx 10^{19}$ $\mathrm{cm}^{-2}$. That is why $\Delta t_{g r}-\mathscr{R} / c \gtrsim 10^{16} / \nu^{2}$ and the delay of pulses at the frequency $v \approx 10^{8} \mathrm{~Hz}$ as compared with the pulses at high frequencies exceeds one second and for a number of pulsars it reaches several minutes.

Inhomogeneities of the interstellar medium should naturally cause fluctuations in the intensity of the radio emission of discrete sources recorded at the Earth (the picture corresponds to the diffraction on the phase screen and its change with the time is determined mainly by the size of inhomogeneities and the relative velocity of the screen and the Earth). The potentialities of corresponding observations had been discussed [87] before the discovery of pulsars but only with the use of pulsar radiation did they become real and are being carried out now [88, 89]. Some contribution to the fluctuations are, of course, due to the corona of the pulsar itself [88-89a] as well as to the interplanetary medium. The contribution of the latter may be comparatively confidently excluded or, on the contrary, we may use pulsars for the investigations of the solar supercorona [90].

The field vector in the linearly polarized wave travelling in the interstellar medium rotates over an angle (see (30) and [49])

$$
\Psi=\frac{\omega}{2 c} \int_{0}^{\mathscr{T}}\left(\tilde{n}_{-}-\tilde{n}_{+}\right) \mathrm{d} s=\frac{2.4 \times 10^{4}}{v^{2}} \int_{0}^{\mathscr{R}} n_{e}(s) H(s) \cos \theta(s) \mathrm{d} s .
$$


Instead of $\Psi$ the rotation measure RM is often used which is defined as the factor in the relation $\Psi=R M \lambda^{2}$, where $R M=8.1 \times 10^{5} \int n_{e} H \cos \theta \mathrm{d} s \operatorname{rad~m^{-2}}$ if $\Psi$ is measured in radians, $\lambda$ in meters, the distance in parsec, $n_{e}$ in $\mathrm{cm}^{-3}$ and $H$ in Oersteds. Thus the knowledge of the angle $\Psi$ allows us to determine the value of $\int_{0}^{\Re} n_{e} H \cos \theta \mathrm{d} s$. The use of pulsars for this purpose is particularly valuable, for at the same time $\mathscr{N}_{e}=\int n_{e} \mathrm{~d} s$ is measured along the same line of sight and, therefore, a mean value $\overline{H \cos \theta}=\int n_{e} H \cos \theta \mathrm{d} s / \int n_{e} \mathrm{~d} s$ is determined for the same line (see [91]). The environment ('corona') of a pulsar has a stronger influence on the estimate of $\bar{H} \overline{\cos \theta}$ for the interstellar medium than on the determination of the mean particle density $\bar{n}_{e}$ (near the pulsar both the values of $n_{e}$ and $H$ increase; besides, the value of $H \cos \theta$ as distinct from $n_{e}$ can change sign). The consideration of the influence of pulsar environments on the polarization of their radiation requires a special analysis [48, $47,92]$; the same may be said about the influence on the polarization of fluctuations of the quantity $n_{e} H \cos \theta$ and also about the determination of this quantity near the Sun (see $[92,93])$. Nevertheless, the use of pulsars for estimations of the longitudinal component $H \cos \theta$ of the magnetic field $\mathbf{H}$ on the line of sight together with the possibility of determining a mean electron concentration $\bar{n}_{e}$ and its fluctuations belongs to the most important applications of pulsar observations for astronomical purposes. Pulsars can be used also in classical astronomy and astrometry [94, 95].

While passing near the Sun the electromagnetic pulse undergoes two closely connected effects of general relativity, i.e. a deflection and an extra delay reaching $2 \times 10^{-4} \mathrm{sec}$ when the ray is grazingly passing the Sun's disk. Due to the latter effect we have to observe an annual change of the period of pulsars located on the celestial sphere near the path of the Sun [94, 96]. However, checking-up general relativity though the observation of an artificial planet with a transmitter on board seems much more hopeful for this purpose.

The light pulses from pulsar NP 0532 arrive simultaneously (accuracy $\approx 10^{-5} \mathrm{sec}$ ) for a number of wave lengths for which the observations have been carried out. Hence, we may come to the conclusion that the velocity of light in this diaphason does not depend on the frequency with a very high degree of accuracy $\left(\Delta c / c<5 \times 10^{-18}\right.$; see [97]). Some other possibilities are discussed in the literature [94, 98] offering the application of pulsars in the investigations of astronomical and physical questions.

\section{Concluding Remarks}

One may think that the content of the report confirms the assertion made at the beginning that the theoretical notions about pulsars and their corresponding models are not at all complete. At the same time progress in the theory of pulsars is obvious and, in particular, several concrete tasks and questions requiring theoretical investigations are already clear. There is no need to enumerate these tasks here as they are partially clear from the above and because I should like to finish the report with more general remarks.

The discovery of pulsars is the last from the five brilliant astronomical discoveries 
made during the previous decade. The other discoveries made within the period 1960-1967 were quasars, cosmic X-ray sources ('X-ray stars'), the relict microwave radiation with a temperature of $2.7 \mathrm{~K}$ and the cosmic masers on the lines of $\mathrm{OH}$, $\mathrm{H}_{2} \mathrm{O}$ and some other molecules. I should note that, though physicists produced a great deal within the same period, their discoveries of comparable importance are perhaps only two, that is the proof of existence of two types of neutrinos (muon and electron neutrinos) and the discovery of CP nonconservation in weak interactions. In this respect we may say that astronomy went ahead of physics but, of course, this was possible only as a result of the use in astronomy of new physical techniques (reception of radio waves, detecting of X-rays and so on). In other words the stream of astronomical discoveries is mainly a manifestation of the process of transformation of astronomy from an optical astronomy to an all-wave one. This process which began after the second World War is likely to be finished during the next decade.

All this is well known but here I should like to emphasize the following: none of the new astronomic discoveries, as far as we know, has exceeded the limits of the known physical laws, or has made us revise or change something in the foundation of physics. Besides, some of newly discovered objects and phenomena were long ago predicted "on the edge of the pen". This refers particularly to neutron stars [81, 99].

Thus not at all belittling the great discoveries made lately by astronomers we may state that this progress has not yet overstepped the limits of astronomy and at any rate in the opinion of the majority of astronomers and physicists it has not put any new principle problems before physics.

Will this situation remain further and, generally, what discoveries or changes of a principle character can be expected in astronomy in the visible future?

It would be most prudent not to put this question at all, for the prophets (or, more prosaically, the prognosticators) have only one common feature, that is to make mistakes or, at least, to make partial mistakes.

But without pretending for some non-trivial prognosis, we may point out some possibilities that have already been discussed*.

In the nearest future we may expect the detection of neutrinos from the Sun. We have also real hopes concerning the recording of neutrinos formed during the flashes of supernovae (i.e., probably in the process of the formation of neutron stars and at the same time of pulsars). So valuable information will be received not only of astronomical character but relating to the physics of neutrinos and generally weak interactions $[100,101]$. More distant seems the possibility of observing neutrinos of relict origin formed during the early stages of cosmical evolution for a number of cosmological models discussed at the present time.

Thus, one of the branches of tomorrow's astronomy is neutrino astronomy.

The idea of gravitational waves (we mean, of course, waves in vacuum) appeared more than half a century ago together with appearance of the theory of general

\footnotetext{
* We put aside the question of the origin of the solar system, the structure of the Moon and planets and so on, together with the problem of the discovery of life or civilization outside the Earth.
} 
relativity (the formula for the intensity of gravitational radiation was obtained by Einstein in 1918 [102]). But we cannot expect gravitational waves to be discovered even today* mainly because of the very small sensitivity of the corresponding receivers as compared with those for electromagnetic waves. We may believe, nevertheless, that one should succeed in receiving gravitational radiation from binary stars and, perhaps, from pulsars during our century (the thirty years that we leave for this should not seem to be too long a period considering that the gravitational waves have already been waiting to be discovered for more than $50 \mathrm{yr}$ ).

The reception of cosmic gravitational waves will make the contents of the 'gravitational waves astronomy' and can bring some unexpectedness (such an unexpectedness could be the discovery of waves with an intensity as was indicated in the experiments [38]; see also [39]).

Within the limits of general relativity the gravitational waves should be strictly transversal. In the tensor-scalar theory for the gravitational field [103] gravitational waves on the contrary have a longitudinal component (parallel to the wave vector). Nevertheless, it is difficult to expect that the fate of the tensor-scalar theory should be decided by studying gravitational waves. It is much more probable that this would be done in the nearest future as a result of more accurate measurements of the deflection of light rays near the Sun or of the time of delay for radio signals passing near to it.

The majority of physicists including the present author are sure of the correctness of general relativity, at any rate for not too strong gravitational fields. But there is also no doubt that further experimental verification of this theory even for weak fields is necessary. After the discovery of the flattening of the Sun [103] the situation in this field even became dramatic. If it turned out that general relativity needed some modification already for weak gravitational fields (within the limits of the solar system; and, specifically, some scalar gravitational field exists) it would be a scientific discovery of greatest importance. In this case, so long as we speak of the use of astronomical measurements, we should really say that astronomy has once more rendered physics an incalculable service.

The possibility that the physical laws and theories already known to us may prove not to be correct increases with the transition to larger and larger spatial and time scales and to larger and larger masses and densities of matter. This refers both to general relativity and to the physics of elementary particles (specifically we mean the conservation of baryon charge and other laws of conservation).

As is known a number of astronomers have already suggested that in the Universe the number of baryons is not conserved (the creation of matter in steady-state cosmology and so on), that the equations of the general relativity are violated for strong fields (for example, in the case of a gravitational collapse) [104], that super-

* If the receivers for gravitational radiation that were used in [38] really recorded gravitational radiation the power of cosmic gravitational radiation is colossal, which seems improbable. For this reason and mainly because of the absence of a number of control experiments the question of the nature of the events observed in [38] cannot yet be considered as settled. 
massive and very dense but sometimes active protobodies exist in stars and particularly in the nuclei of galaxies [105-108] and so on. The steady-state cosmology seems now practically rejected but in the other cases mentioned things are far from clear. The present author is a supporter of a 'healthy conservatism' i.e. does not see any reason for supporting new fundamental ideas before the appearance of cogent arguments in their favour. To my mind there are no such arguments at present. But the very problem of the search for new fundamental ideas and opinions in astronomy (including cosmology) undoubtedly does not only exist but from a certain point of view it is the most interesting one. A concrete prognosis in such cases as a matter of fact is impossible.

Are all these remarks justified, however, in a report on pulsars? We see this justification in the fact that all the mentioned (and, practically, all known to us) trends of succeeding astronomic investigations of a fundamental character are directly or indirectly connected with neutron stars and, therefore, with pulsars! As a matter of fact, it is precisely the neutron stars that belong to the number of most intensive potential sources of cosmic neutrino and gravitational waves. Of all known stars relativistic effects are particularly strong for neutron stars and, thus, the question of the applicability of general relativity is here of particular importance. Finally, the central density of neutron stars is the highest for all known (but not only hypothetical) objects. That is why if a 'new' physics proves to be necessary it would not, probably, go past the neutron stars.

Thus pulsars are not only in the focus of present-day astronomy but, in all probability, they will stay in the centre of attention for many years and even decades.

\section{Acknowledgements}

In the preparation and editing of the present report the author has taken advice from a number of his colleagues in the U.S.S.R. and in other countries. I am sincerely grateful to all of them.

Notes Added in Proof. Let us mention briefly some new results.

(1) Polarization measurements in the radioregion for four pulsars [109] and apparently those [110] for optical radiation of pulsar NP 0532 testify to the model in which the polarization diagram of pulsar radiation is 'pencil-beam' with the axis close to the magnetic one. It is also mentioned in paper [110] that the differences between polarization and some other characteristics of NP 0532 radiation in opticand radioregions indicate the different mechanisms of optical and radio emission.

The latter conclusion seems well enough grounded but to the same degree it follows already from the general considerations presented in Sections 5 and 6 of the present paper.

About the polar diagram and the factors defining its width (the beaming mechanism) see Ref. 111 and 112 .

(2) We should mention papers $[113,114]$ devoted to pulsar magnetosphere and 
paper [115] referring to pulsar dynamics with the account taken of its nonspherical shape.

(3) It is expedient to distinguish between the outer and inner layers of the crust [116]. There are practically no free neutrons in the outer layer whereas the inner one is just characterized by the presence of free neutrons. The corresponding boundary lies at the density $\varrho \approx 3.10^{11} \mathrm{~g} \cdot \mathrm{cm}^{-3}$. When the density increases, the number of neutrons naturally grows and at the density $\varrho \approx 10^{14} \mathrm{~g} \cdot \mathrm{cm}^{-3}$ the crust vanishes rather rapidly (in the density scale). As was shown in Section 3 of the present paper the neutron liquid formed (with the admixture of proton and electron liquids) is apparently superfluid.

However, the neutrons in the inner part of the crust (i.e. at the densities $3.10^{11} \lesssim$ $\lesssim \varrho \lesssim 10^{14}$ or in somewhat narrower density interval) probably also form a superfiuid subsystem.

(4) The question of the state and structure of the substance of the part of the outer crust layer close to the star surface is not yet clear. In this region it is necessary, generally speaking, to take into account the influence of the magnetic field which can lead to the formation of original molecular and quasipolymer structures [117].

In this connection the use of estimate (2) for melting temperature $T_{m}$ near the crust surface is unlikely to be justified. Besides, some thin layer (atmosphere) of gas or, better to say, fluid plasma is apparently to be formed over the crust. Characteristics of this layer (in particular its chemical composition) seem rather essential from the point of view of the conditions of plasma outflow from the star, and therefore for the understanding of the processes in a neutron star magnetosphere.

\section{References*}

[1] Hewish, A., Bell, S. J., Pilkington, J. D. H., Scott, P. F., and Collins, R. A.: 1968, Nature 217, 709.

[2] Ostriker, J. P.: 1968, Nature 217, 1227.

[3] Thorne, K. S.: 1969, Comments Astrophys. Space. Phys. 1, 12.

[4] Ostriker, J. P. and Tassoul, J. L.: 1968, Nature 219, 577; 1969, Astrophys. J. 155, 987.

[5] Wang, C. G., Rose, W. K., and Schlenker, S. L.: 1970, Astrophys. J. (Letters) 160, L17.

[6] Cameron, A. G. W. and Cohen, J. M.: 1969, Astrophys. Letters 3, 3; see also 1970, Astrophys. Space Sci. 6, 228.

Cameron, A. G. W.: 'Neutron Stars', 1970, Ann. Rev. Astron. Astrophys. 8, 179.

[7] Gold, T.: 1968, Nature 218, 731; 1969, 221, 25.

[8] Ginzburg, V. L.: 1964, Dokl. Akad. Nauk SSSR 156, 43 (English transl. Soviet Phys,-Doklady). Ginzburg, V. L. and Ozernoy, L. M.: 1964, Zh. Exper. Teor. Fiz. 47, 1031 (English transl. Soviet Phys.-JETP). Ozernoy, L. M.: 1968, Highlights of Astronomy (ed. by L. Perek), Reidel, Dordrecht-Holland, p. 384. Ozernoy, L. M. and Chertoprud, V. E.: 1969, Astron. Zh. 46, 940.

[8a] Morrison, P. 1969, Astrophys. J. (Letters) 157, L73.

[9] Layzer, D.: 1968, Nature 220, 247.

[10] Hoyle, F. and Narlikar, J.: 1968, Nature 218, 123.

[11] Harrison, E. R.: 1970, Nature 225, 44.

* The bibliography given is far from being complete. In the part of the Commission 40 IAU Draft Report 1970 devoted to pulsars (prepared by S. P. Maran) 100 references are given and it is indicated that on September 1st 1969 nearly 330 papers were connected with pulsars. By August 1970 the number of such papers will probably reach about 500 . 
[12] Arnett, W. D.: 1969, Nature 222, 359.

Cameron, A.G.W.: 1969, Comments Astrophys. Space Phys. 1, 172.

[13] Woltjer, L.: 1964, Astrophys. J. 140, 1309.

[13] Pacini, F.: 1967, Nature 216, 567.

[15] Ruderman, M.: 1968, Nature 218, 1128.; 1969, Report 6/69, New York University, Department of Physics.

[16] Nemeth, J. and Sprung, D. W. L.: 1968, Phys. Rev. 176, 1496.

[17] Ginzburg, V. L. and Kirzhnits, D. A.: 1964, Zh. Exper. Teor. Fiz. 47, 2006. (English transl. Soviet Phys.-JETP 20, 1346 (1965)). Ginzburg, V. L.: 1969, J. Stat. Phys. 1, 3; 1969, Comments Astrophys. Space Phys. 1, 81.

[18] Migdal, A. B.: 1959, Zh. Exper. Teor. Fiz. 37, 249.

[19] Wolf, R. A.: 1966, Astrophys. J. 145, 834.

[20] Baym, G., Pethick, C., and Pines, D.: 1969, Nature 224, 673.

[21] Itoh, N.: 1969, Progress Theor. Phys, 42, 1478.

[22] Hoffberg, M., Glassgold, A. E., Richardson, R. W., and Ruderman, M.: 1970, Phys. Rev. Letters 24, 775.

[23] Pearson, J. M. and Saunier, G.: 1970, Phys. Rev. Letters 24, 325.

[24] Canuto, V., Chiu, H. Y., Chiuderi, C., and Lee, H. J.: 1970, Nature 225, 47.

[25] Solinger, A.: 1969, preprint.

[26] Baym, G., Pethick, C., and Pines, D.: 1969, Nature 224, 674.

[27] Gunn, J. E. and Ostriker, J. P.: 1970, Astrophys. J. 160, 979; 1969, Nature 223, 813.

[27a] Setti, G. and Woltjer, L.: 1970, Astrophys. J. (Letters) 159, L87.

[28] Ruderman, M.: 1969, Nature 223, 597. Smoluchowski, R.: 1970, Phys. Rev. Letters 24, 923.

[29] Dyson, F. J.: 1969, Comments Astrophys. Space Phys. 1, 198.

[30] Baym, G., Pethick, C., Pines, D., and Ruderman, M.: 1969, Nature 222, 872.

[31] Sutherland, P., Baym, G., Pethick, C., and Pines, D.: 1970, Nature 225, 353.

[32] Ruderman, M.: 1970, Nature 225, 619.

[33] Michel, F. C.: 1970, Astrophys. J. (Letters) 159, L25.

[34] Ruderman, M.: 1970, Nature 225, 838.

[34a] Schvarzman, V. F.: 1970, Astron. Circ. N 563.

[34b] Bisnovaty-Kogan, G. S.: 1970, Astron. Circ. U.S.S.R. N 529.

[35] Deutsch, A. J.: 1955, Ann. Astrophys. 18, 1.

[36] Ostriker, J. P. and Gunn, J. E.: 1969, Astrophys. J. 157, 1395.

[37] Melosh, H. J.: 1969, Nature 224, 781.

[38] Weber, J.: 1969, Phys. Rev. Letters 22, 1320; 1970, ibid. 24, 276.

[39] Braginskii, V. B. and Rudenko, V. N.: 1970, Uspekhi Fiz. Nauk 100, 395.

[40] Fairbank, W. M.: 1969, private communication.

[41] Cavaliere, A. and Pacini, F.: 1970, Astrophys. J. (Letters) 159, L21.

[42] Goldreich, P. and Julian, W. H.: 1969, 157, 869. Astrophys. J.

[43] Michel, F. C.: 1969, Phys. Rev. Letters 23, 247; 1969, Astrophys. J. 727.

[44] Davis, L.: 1947, Phys. Rev. 72, 632.

[45] Mestel, L.: 1968, Monthly Notices Roy. Astron. Soc. 140, 177; preprint, 1969.

[46] Occhionero, F. and Demianski, M.: 1969, Phys. Rev. Letters 23, 1128.

[47] Schvarzman, V. F.: 1970, Radiofisica, in press.

[48] Ginzburg, V. L., Zheleznyakov, V. V., and Zaitsev, V. V.: 1969, Astrophys. Space Sci. 4, 464; 1968, Nature 220, 355; 1969, 222, 230.

[49] Ginzburg, V. L.: 1964, The Propagation of Electromagnetic Waves in Plasmas, Pergamon Press; revised edition, 1970.

[50] Dokuchaev, V. P.: 1970, Astrofisica 6, 471.

[51] Michel, F. C. and Goldwire, H. C.: 1970, Astrophys. Letters 5, 21.

[52] Davis, L. and Goldstein, M.: 1970, Astrophys. J. (Letters) 159, L81.

[52a] Goldreich, P.: 1970, Astrophys. J. (Letters) 160, L11.

[53] Ginzburg, V. L.: 1943, Phys. Rev. 63, 1; 1944, J. Phys. U.S.S.R. 8, 33.

[54] Böhm-Vitense, E.: 1969, Astrophys. J. (Letters) 156, L131.

[55] Wampler, E. J., Scargle, J. D., Miller, J. S.: 1969, Astrophys. J. (Letters) 157, L1.

[56] Preston, G. W.: 1967, Astrophys. J. 150, 547; Landstreet, J. D.: 1970, Astrophys. J. 159, 1001.

[57] Piddington, J. H.: 1969, Nature 222, 965. 
[58] Pacini, F.: 1970, 'Neutron Stars, Pulsar Radiation and Supernova Remnants', preprint; Bertotti, B., Cavaliere, A., and Pacini, F.: 1969, Nature 223, 1351.

[59] Ginzburg, V. L.: 1969, Comments Astrophys. Space Phys. 1, 207.

[60] Scargle, J. D. and Harlan, E. A.: 1970, Astrophys. J. (Letters) 159, L143.

[61] Ginzburg, V. L. and Zheleznyakov, V. V.: 1969, Uspekhi Fiz. Nauk 99, 514, 524 (English transl. Soviet Phys.-Uspekhi).

[62] Ginzburg, V. L. and Zheleznyakov, V. V.: 1970, Comments Astrophys. Space Phys. 2, 167, 197.

[63] Ginzburg, V. L. and Syrovatskii, S. 1.: 1969, Ann. Rev. Astron. Astrophys. 7, 375.

[64] Zheleznyakov, V. V.: 1970, Radio Emission from Sun and Planets, Pergamon Press.

[65] Kaplan, S. A. and Tsytovich, V. N.: 1969, Uspekhi Fiz. Nauk 97, 77 (English transl. Soviet Phys.Uspekhi).

[66] Ginzburg, V. L.: 1959, Uspekhi Fiz. Nauk 69, 537. (English transl. Soviet Phys.-Uspekhi 2 (1960), 874 .

[67] Ginzburg, V. L. and Syrovatskii, S. I.: 1965, Ann. Rev. Astron. Astrophys. 3, 297.

[68] Chiu, H. Y. and Canuto, V.: 1969, Phys. Rev. Letters 22, 415; 1969, Nature 221, 529; 1969, 223, 1113.

[69] Papagiannis, M. D.: 1969, Nature 222, 1261.

[70] Radhakrishnan, V. and Cooke, D. J.: 1969, Astrophys. Letters 3, 225.

[71] Shklovsky, I. S.: 1969, Uspekh. Fiz. Nauk 99, 526.

[72] Apparao, K. M. V. and Hoffman, J.: 1970, Astrophys. Letters 5, 25.

[73] Kaplan, S. A. and Tsytovich, V. N.: 1969, Symposium report (Rome, Dec.) and preprint (1970).

[73a] Ichimaru, S.: 1970, Nature 226, 731.

[74] Shklovsky, I. S.: 1970, Nature 225, 251; 1970, Astrophys. J. (Letters) 1970, 159, L77; see also 161, L63.

[75] Getmansev, G. G. and Ginzburg, V. L.: 1952, Dokl. Akad. Nauk S.S.S.R. 87, 187.

[76] Komesaroff, M. M.: 1970, Nature 225, 612.

[77] Zheleznyakov, V. V.: 1970, Radiofisica 13, 1892.

[77a] Drake, F. D. and Craft, H. D.: 1968, Nature 220, 231 ; Taylor, J. H., Jura, M., and Huguenin, G. R.: 1969, Nature 223, 797; Vitkevich, V. V. and Shitov, Yu. P.: 1970, Nature 225, 248; Sutton, J. M., Staelin, D. H., Price, R. M., Weimer, R.: 1970, Astrophys. J. (Letters) 159, L89.

[78] Holt, S. S. and Ramaty, R.: 1970, Astrophys. Letters. 5, 89.

[79] Lunds, R.: 1969, Astrophys. J. (Letters) 157, L11.

[80] Greenstein, J. L.: 1970, private communication.

[81] Baade, W. and Zwicky, F.: 1934, Proc. Nat. Acad. Sci. Amer. 20, 259.

[82] Tucker, W. H.: 1969, Nature 223, 250.

[83] Ostriker, J. P.: 1970, Proc. Ilth Intern. Conf. on Cosmic Rays, Budapest, in press.

[84] Apparao, K. M. V. and Rengarajan, T. N.: 1969, 'Pulsars and Cosmic Rays', preprint.

[85] Pollack, J. B. and Shen, B. S. P.: 1969, Phys. Rev. Letters 23, 1358.

[85a] Ginzburg, V. L. and Erukhimov, L. M.: 1971, Astrophys. Space Sci., in press.

[86] Prentice, A. J. R. and ter Haar, D.: 1969, Monthly Notices Roy. Astron. Soc. 146, 423.

[87] Ginzburg, V. L.: 1956, Dokl. Akad. Nauk S.S.S.R. 109, 61; Pisareva, V. V.: 1958, Astron. Zh. 35, 112.

[88] Erukhimov, L. M. and Pisareva, V. V.: 1968, Astron. Circ. S.S.S.R. N 489; 1969, Radiofisica 12, 900; Erukhimov, L. M.: 1969, Astron. Circ. S.S.S.R. N 513.

[89] Scheuer, P. A. G.: 1968, Nature 218, 970; Salpeter, E. E.: 1969, Nature 221, 31; Rickett, B. J.: 1969, Nature 221, 158; Lang, K. R.: 1969, Science 166, 1401.

[89a] Code, A. D.: 1970, Astrophys. J. 159, L29.

[90] Holweg, J. V.: 1968, Nature 220, 771; Goldstein, S. J. and Meisel, D. D.: 1969, Nature 224, 349.

[91] Smith, F. G.: 1968, Nature 218, 325; 1968, 220, 891; Ekers, R. D., Lequeux, J., Moffet, A. T., and Seielstad, G. A.: 1969, Astrophys. J. (Letters) 156, L21; Staelin, D. H. and Reifenstein, D. H.: 1969, Astrophys. J. (Letters) 156, L121.

[92] Lotova, N. A.: 1969, Astron. Zh. 46, 1165.

[93] Ginzburg, V. L. and Pisareva, V. V.: 1963, Radiofisica 6, 877; Ginzburg, V. L.: 1960, Radiofisica 3, 341 .

[94] Counselman, C. C. and Shapiro, I. I.: 1968, Science 162, 352.

[95] Martynov, D. Ya.: 1969, Astron. Circ. S.S.S.R. N 512, 513; Maran, S. P. and Ogelman, H.: 1969, Nature 224, 349. Pfleiderer, J.: 1970, Nature 225, 437. 
[96] Richard, J. P.: 1968, Phys. Rev. Letters 21, 1483.

[97] Warner, B. and Nather, R. E.: 1969, Nature 222, 157.

[98] Feinberg, G.: 1964, Science 166, 879.

[99] Landau, L. D.: 1938, Nature 141, 333.

[100] Zeldovich, Ya. B.: 1970, Comments Astrophys. Space Phys. 2, 12.

[101] Stothers, R. B.: 1970, Phys. Rev. Letters 24, 538.

[102] Einstein, A.: 1918, Sitzungsber. preuss. Akad. Wiss. 1, 154.

[103] Dicke, R. H.: 1962, Phys. Rev. 125, 2163; 1970, Astrophys. J. 159, 1.

[104] Hoyle, F.: 1948, Monthly Notices Roy. Astron. Soc. 108, 372; 1949, 109, 365; 1969, J. Roy. Astron. Soc. 10, 10; Hoyle, F. and Narlikar, J. V.: 1963, Proc. Roy. Soc. 273, 4; 1966, 294, 138.

[105] Jeans, J.: 1928, Astronomy and Cosmogony, Cambridge University Press, Cambridge, p. 352.

[106] Ambartsumian, V.: 1965, The Structure and Evolution of Galaxies (Proc. 13 Solvay Conference on Physics), Interscience Publishers, New York, p. 1.

[107] Burbidge, G. R.: 1970, Astrophys. J. (Letters) 159, L105.

[108] Low, F. J.: 1970, Astrophys. J. (Letters) 159, L173.

[109] Manchester, R. N.: 1970, Nature 228, 264.

[110] Cocke, W. J., Disney, M. J., Muncaster, G. W., and Gehrels, T.: 1970, Nature 227, 1327.

[111] Smith, F. G.: 1970, Nature 228, 913; 1970, Monthly Notices Roy. Astron. Soc. 149, 1.

[112] Zheleznyakov, V. V.: 1971, Astrophys. Space Sci., in press.

[113] Endean, V. G. and Allen, J. E.: 1970, Nature 228, 348.

[114] Michel, F. C.: 1970, Comments Astrophys. Space Phys. 2, 227.

[115] Burns, J. A.: 1970, Nature 228, 986.

[116] Pines, D.: 1970, Preprint, Nordita.

[117] Kadomtsev, B. B.: 1971, Uspekhi Fiz. Nauk; see also Cohen, R., Lodenquai, J., and Ruderman, M.: 1970, Phys. Rev. Letters 25, 467. 
[96] Richard, J. P.: 1968, Phys. Rev. Letters 21, 1483.

[97] Warner, B. and Nather, R. E.: 1969, Nature 222, 157.

[98] Feinberg, G.: 1964, Science 166, 879.

[99] Landau, L. D.: 1938, Nature 141, 333.

[100] Zeldovich, Ya. B.: 1970, Comments Astrophys. Space Phys. 2, 12.

[101] Stothers, R. B.: 1970, Phys. Rev. Letters 24, 538.

[102] Einstein, A.: 1918, Sitzungsber. preuss. Akad. Wiss. 1, 154.

[103] Dicke, R. H.: 1962, Phys. Rev. 125, 2163; 1970, Astrophys. J. 159, 1.

[104] Hoyle, F.: 1948, Monthly Notices Roy. Astron. Soc. 108, 372; 1949, 109, 365; 1969, J. Roy. Astron. Soc. 10, 10; Hoyle, F. and Narlikar, J. V.: 1963, Proc. Roy. Soc. 273, 4; 1966, 294, 138.

[105] Jeans, J.: 1928, Astronomy and Cosmogony, Cambridge University Press, Cambridge, p. 352.

[106] Ambartsumian, V.: 1965, The Structure and Evolution of Galaxies (Proc. 13 Solvay Conference on Physics), Interscience Publishers, New York, p. 1.

[107] Burbidge, G. R.: 1970, Astrophys. J. (Letters) 159, L105.

[108] Low, F. J.: 1970, Astrophys. J. (Letters) 159, L173.

[109] Manchester, R. N.: 1970, Nature 228, 264.

[110] Cocke, W. J., Disney, M. J., Muncaster, G. W., and Gehrels, T.: 1970, Nature 227, 1327.

[111] Smith, F. G.: 1970, Nature 228, 913; 1970, Monthly Notices Roy. Astron. Soc. 149, 1.

[112] Zheleznyakov, V. V.: 1971, Astrophys. Space Sci., in press.

[113] Endean, V. G. and Allen, J. E.: 1970, Nature 228, 348.

[114] Michel, F. C.: 1970, Comments Astrophys. Space Phys. 2, 227.

[115] Burns, J. A.: 1970, Nature 228, 986.

[116] Pines, D.: 1970, Preprint, Nordita.

[117] Kadomtsev, B. B.: 1971, Uspekhi Fiz. Nauk; see also Cohen, R., Lodenquai, J., and Ruderman, M.: 1970, Phys. Rev. Letters 25, 467. 